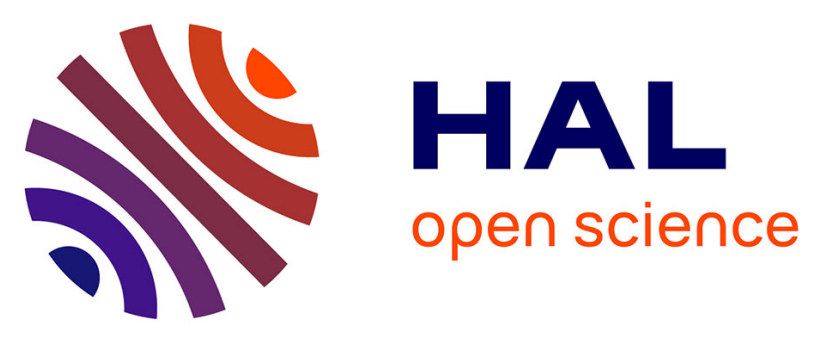

\title{
A new chimeric triple reporter fusion protein as a tool for in vitro and in vivo multimodal imaging to monitor the development of African trypanosomes and Leishmania parasites
}

Estefania Calvo-Alvarez, Christelle Cren-Travaillé, Aline Crouzols, Brice Rotureau

\section{To cite this version:}

Estefania Calvo-Alvarez, Christelle Cren-Travaillé, Aline Crouzols, Brice Rotureau. A new chimeric triple reporter fusion protein as a tool for in vitro and in vivo multimodal imaging to monitor the development of African trypanosomes and Leishmania parasites. Infection, Genetics and Evolution, 2018, 10.1016/j.meegid.2018.01.011 . pasteur-01849818

\section{HAL Id: pasteur-01849818}

\section{https://hal-pasteur.archives-ouvertes.fr/pasteur-01849818}

Submitted on 3 Aug 2018

HAL is a multi-disciplinary open access archive for the deposit and dissemination of scientific research documents, whether they are published or not. The documents may come from teaching and research institutions in France or abroad, or from public or private research centers.
L'archive ouverte pluridisciplinaire HAL, est destinée au dépôt et à la diffusion de documents scientifiques de niveau recherche, publiés ou non, émanant des établissements d'enseignement et de recherche français ou étrangers, des laboratoires publics ou privés. 


\section{A new chimeric triple reporter fusion protein as a tool for in vitro}

2 and in vivo multimodal imaging to monitor the development of

3 African trypanosomes and Leishmania parasites.

4

5 Estefania Calvo Alvarez*, Christelle Cren-Travaillé, Aline Crouzols and Brice Rotureau 6

7 Trypanosome Transmission Group, Trypanosome Cell Biology Unit, Institut Pasteur and 8 INSERM U1201, Paris, France

9 * Corresponding author: Estefania Calvo Alvarez, estefania.calvo-alvarez@pasteur.fr

11 Key words: Trypanosoma, Leishmania, bioluminescence, fluorescence, intravital imaging, 12 fusion protein, reporter.

Short title: A triple reporter for multimodal imaging of trypanosomatids

16 Number of figures: $8(+3)$

\section{Highlights}

- New triple reporter combining a red luciferase and a tdTomato fused by a Ty1-tag

19 - Tested in two different stages of both T. brucei and L. major parasites

20 - Tested in vitro and in vivo in tsetse flies and murine models

21 - Useful for multimodal imaging at different scales, including intravital monitoring 


\section{Abstract}

24 Trypanosomiases and leishmaniases, caused by a group of related protist parasites, are

25 Neglected Tropical Diseases currently threatening more than 500 million people worldwide.

26 Reporter proteins have revolutionised the research on infectious diseases and have opened

27 up new advances in the understanding of trypanosomatid-borne diseases in terms of both

28 biology, pathogenesis and drug development. Here, we describe the generation and some

29 applications of a new chimeric triple reporter fusion protein combining the red-shifted firefly

30 luciferase PpyREH9 and the tdTomato red fluorescent protein, fused by the TY1 tag.

31 Expressed in both Trypanosoma brucei brucei and Leishmania major transgenic parasites,

32 this construct was successfully assessed on different state-of-the-art imaging technologies,

33 at different scales ranging from whole organism to cellular level, both in vitro and in vivo in

34 murine models. For $T$. b. brucei, the usefulness of this triple marker to monitor the entire

35 parasite cycle in both tsetse flies and mice was further demonstrated. This stable reporter

36 allows to qualitatively and quantitatively scrutinize in real-time several crucial aspects of the

37 parasite's development, including the development of African trypanosomes in the dermis of

38 the mammalian host. We briefly discuss developments in bio-imaging technologies and

39 highlight how we could improve our understanding of parasitism by combining the genetic

40 engineering of parasites to the one of the hosting organisms in which they complete their

41 developmental program. 
Vector-borne diseases affect about half of the World's population and are endemic in more than 100 countries. Among these vector-borne diseases, trypanosomiases and leishmaniases are considered as important Neglected Tropical Diseases (NTDs) that especially impact public health, accounting for $\sim 24$ million persons infected worldwide among over 500 million people at risk. These diseases are (i) Human African Trypanosomiasis (HAT) or sleeping sickness that is caused by the African trypanosomes Trypanosoma brucei (Buscher et al., 2017; Franco et al., 2017), (ii) Chagas disease or American Trypanosomiasis that is due to the American trypanosome Trypanosoma cruzi (Perez-Molina and Molina, 2017; Stanaway and Roth, 2015), and (iii) leishmaniases, a group of visceral and cutaneous diseases caused by a wide range of Leishmania spp. (Alvar et al., 2012; Matlashewski et al., 2014; Reithinger et al., 2007). In addition, some other trypanosome species (mainly $T$. evansi, $T$ equiperdum, $T$. congolense and $T$. vivax) cause similar diseases in domestic animals and are responsible for important economic losses in developing countries (Rotureau and Van Den Abbeele, 2013). There is no vaccine against these parasites, most of the few currently available medications are not easily administrable and frequently present severe side effects, and drug resistance is dramatically increasing. However, in addition to mass screening and treatment programs, strategies for reservoir and / or vector control have proven their efficiency in certain endemic areas (Aksoy et al., 2017; Dias, 2015; Gonzalez et al., 2015). In vivo intravital imaging methods have tremendously been improved over the last decades, and now represent powerful tools for the understanding of a large panel of dynamic biological processes, especially in the field of infectious diseases (Coombes and Robey, 2010). Beyond their obvious contribution to the ethical improvement of the experimental design of research studies on living animals, especially in terms of reduction and refinement, these approaches also offer a powerful and almost unbiased mean to monitor and compare the variable kinetics of infection among individuals. In the context of diagnostic tool and drug development, as well as in order to study the complex developmental cycles of trypanosomatid parasites, genetically engineered parasites have been developed to express a large panel of molecular reporters including fluorescent and bioluminescent probes both in T. cruzi (Lewis et al., 2015) and T. brucei (Burrell-Saward et al., 2015; Coles et al., 2017; MacLean et al., 2013; McLatchie et al., 2013; Van Reet et al., 2014), and especially in Leishmania spp. (Beattie and Kaye, 2011; Calvo-Alvarez et al., 2015a; Carneiro et al., 2017; Forestier, 2013; Lang et al., 2009; Taheri et al., 2015).

The choice of a given reporter is conditioned by that of a specific readout methodology that usually focuses on parasite detection at a single scale, i.e. at the cellular level in multi-well plates for drug screening, at the tissue level in ex vivo organ explants or models, at the entire 
organism level to study the kinetics of the infection in lab animal models. However, only few attempts to combine these modes of detection and / or imaging have been proposed, especially in trypanosomatid research (Taheri et al., 2015). From the literature, dual reporter fusion proteins have been designed for the purpose of combining bioluminescence and fluorescence-based approaches: firefly luciferase Luc and EGFP (Patel et al., 2010; Taheri et al., 2015), firefly luciferase Luc2 and EGFP (Liu et al., 2010), firefly luciferase Luc2 and tdTomato (Liu et al., 2010), firefly luciferase Luc2 and TurboFP635 (Mezzanotte et al., 2014), mVenus and Renilla luciferase RLuc8, mTurquoise2 and RLuc8, mKusabiraOrange2 and RLuc8 (Takai et al., 2015). All these constructs encode for at least one marker emitting in the green. This is not optimal for intravital imaging due to the high absorption of the light at these wavelengths by the melanin and haemoglobin proteins present in tissues. To bypass this technical bias, McLatchie and colleagues have first reported a system that allows the detection of fewer than 100 bioluminescent $T$. brucei parasites in a murine model by the use of the codon-optimised red-shifted Photinus pyralis luciferase (PpyRE9H) (McLatchie et al., 2013). They were able to monitor in real time the parasite dissemination and to detect brain infections with a level of sensitivity in vivo that was significantly greater than achievable with a yellow firefly luciferase reporter (McLatchie et al., 2013). Almost at the same time, Van Reet et al. have compared the usefulness of three different red-shifted luciferases, and characterized their in vitro activity and growth profile, as well as their potential for in vivo murine infection follow-up in a set of diverse T. brucei strains (Van Reet et al., 2014). BurrellSaward and co-workers also used the red bioluminescent $T$. b. brucei strain generated by McLatchie et al. to evaluate the efficacy of novel drugs in vivo against late-stage HAT and were able to detect drug relapse earlier than traditional methods (Burrell-Saward et al., 2015). In order to go a step further, we have selected and combined two reporters producing light in the red wavelengths (tdTomato at $581 \mathrm{~nm}$ and red-shifted firefly luciferase PpyREH9 at $617 \mathrm{~nm}$ ) with a reduced absorption in tissues, therefore resulting in an enhanced sensitivity of the monitoring.

106 Common protocols, such as for electron microscopy, histology or protein biochemistry, can require deleterious heat and / or chemical treatments of the samples that frequently result in a denaturation of these recombinant proteins preventing further fluorescent emission, enzymatic activities as well as immuno-detection. To circumvent these obstacles, the Ty1 epitope has become a popular choice in trypanosomes, but also in other parasites such as Leishmania and Toxoplasma (Bastin et al., 1996). The Ty-tag is a 10-amino-acid sequence

112 from the immunologically well-characterized major structural protein of the Ty1 virus-like 113 particle of Saccharomyces cerevisiae (Brookman et al., 1995). The epitope is recognized by 114 two different monoclonal antibodies, BB2 $(\lg G 1)$ and TYG5 $(\lg A)$, that were tested in a 115 variety of immunological conditions and that do not cross-react with trypanosome proteins. 
116 Although a number of commercial antibodies from various companies are available against 117 either firefly luciferase or tdTomato, the Ty1-tag has been used extensively in 118 Trypanosomatids and it has already proved its usefulness in multiple contexts (Huet et al., 119 2014; Julkowska and Bastin, 2009; Ooi et al., 2015). Therefore, between the two red-emitting 120 probes, we have inserted a small Ty1-tag (Bastin et al., 1996) that can be detected in most of 121 conditions, in order to obtain a stable cytosolic triple marker fusion protein 122 (PpyRE9H/TY1/tdTomato) that can be used to study the same sample by multi-modal 123 detection and imaging approaches.

124 Here, we describe the generation of this new chimeric triple reporter fusion protein. This 125 construct was successfully assessed on state-of-the-art imaging technologies both in vitro 126 and in vivo by using Trypanosoma brucei and Leishmania major transgenic parasites. This 127 approach allowed us to scrutinize the development of both parasites within the mammalian 128 host, as well as the journey of African trypanosomes in the tsetse fly digestive tract. 


\subsection{Leishmania major and Trypanosoma brucei brucei strains and culture}

131 The AnTat 1.1E pleomorphic clone of Trypanosoma brucei brucei was derived from a strain

132 originally isolated from a bushbuck in Uganda in 1966 (Le Ray et al., 1977). Procyclic forms

133 (PCF) were grown at $27^{\circ} \mathrm{C}$ in SDM-79 medium (Brun and Schonenberger, 1979)

134 supplemented with $10 \%(\mathrm{v} / \mathrm{v})$ heat-inactivated foetal calf serum (FCS) and $8 \mathrm{mM}$ glycerol

135 (SDMG). Bloodstream forms (BSF) of the same strain were cultivated in complete HMI-11 136 medium (Kooy et al., 1989) at $37^{\circ} \mathrm{C}$ in $5 \% \mathrm{CO}_{2}$. For in vitro slender to stumpy $\mathrm{BSF}$ 137 differentiation, we used 8-pCPT-2'-O-Me-5'-AMP, a nucleotide analog of 5'-AMP from 138 BIOLOG Life Science Institute (Germany). Briefly, $2 \times 10^{6}$ pleomorphic AnTat 1.1E slender 139 forms were incubated with 8-pCPT-2'-O-Me-5'-AMP $(5 \mu \mathrm{M})$ for $48 \mathrm{~h}$ (Barquilla et al., 2012). 140 Freshly differentiated stumpy forms were then centrifuged and resuspended in SDMG 141 medium with $10 \mathrm{mM}$ glutathione for tsetse fly infection.

142 Leishmania major strain Friedlin V1 (MHOM/JL/80/Friedlin) (Kapler et al., 1990), was 143 cultured at $26^{\circ} \mathrm{C}$ in M199 supplemented with $25 \mathrm{mM}$ HEPES pH 7.2, $0.1 \mathrm{mM}$ adenine, $1440.0005 \%(\mathrm{w} / \mathrm{v})$ hemin, $2 \mu \mathrm{g} / \mathrm{ml}$ biopterin, $0.0001 \%(\mathrm{w} / \mathrm{v})$ biotin, 10\% (v/v) FCS and antibiotic 145 cocktail (50 U/ml penicillin, $50 \mu \mathrm{g} / \mathrm{ml}$ streptomycin).

\section{$146 \quad 2.2$ Construction of the chimeric fusion DNA sequences}

$147 \mathrm{~A} \quad 3.1 \mathrm{~Kb}$ sequence coding for the chimeric multiplex reporter protein 148 PpyRE9H/TY1/tdTomato was genetically engineered. The resulting cytoplasmic reporter is 149 composed of three distinct markers: the red-shifted luciferase (PpyRE9H) is fused to the 150 tdTomato red fluorescent protein by a Ty1 tag.

151 To generate RED T. brucei parasites, the $1.68 \mathrm{~Kb}$ optimised version of the North American

152 firefly Photinus pyralis luciferase (Branchini et al., 2005) was fused with a 30-bp sequence 153 known as Ty1-tag (Bastin et al., 1996) and cloned into the pTSARib plasmid (Xong et al., 154 1998) by using Xhol and HindIII restriction enzymes to obtain the pTSARib-PpyRE9H-TY1 155 plasmid. Finally, the $1.4 \mathrm{~Kb}$ coding region of the tdTomato fluorescent protein was inserted 156 downstream using HindIII and BamHI. The resulting $8.9 \mathrm{~Kb}$ pTSARib-PpyRE9H-TY1157 tdTomato vector, containing a blasticidin S resistance cassette, was linearised with Sphl to 158 integrate the rDNA promoter locus.

159 In order to generate the RED L. major strain, the same chimeric PpyRE9H/TY1/tdTomato 160 protein coding sequence was amplified by PCR from the pTSARib-PpyRE9H-TY1-tdTomato 161 vector. PCR product was cut with $\mathrm{Bg} / \mathrm{ll}$ and Notl restriction enzymes and ligated into the 162 same sites of the pLEXSY-hyg2 expression vector (Jena Bioscience GmbH, Germany). 


\subsection{Generation of the RED parasites}

164 Parasites genetically engineered to express the chimeric multiplex reporter protein

165 PpyRE9H/TY1/tdTomato in their cytosol will be further referred as RED strains.

166 Trypanosomes BSF and PCF were transformed with an Amaxa Nucleofector (Lonza)

167 (Burkard et al., 2007; MacGregor et al., 2013) and sub-cloned by limiting dilution. Clone

168 selection was performed by measuring both bioluminescence in a microplate reader Infinite $\AA$

169200 (Tecan) and fluorescence with a Muse ${ }^{\circledR}$ cell Analyzer (Merck-Millipore). Cells were

170 routinely counted with an automated Muse $®$ cell Analyzer (Merck-Millipore) and / or manually

171 with a KOVA hemocytometer according to the manufacturer's recommendations.

172 L. major promastigote parasites were transformed with the large Swal targeting fragment 173 derived from the final $11.1 \mathrm{~Kb}$ pLEXSY-PpyRE9H-TY1-tdTomato plasmid by electroporation

174 and subsequent plating on semisolid media containing $200 \mu \mathrm{g} / \mathrm{ml}$ hygromycin B as previously 175 described (Kapler et al., 1990). The fluorescent and bioluminescent clones were confirmed 176 by using a microplate reader Infinite $\AA 200$ (Tecan) and a Muse $®$ cell Analyzer (Merck177 Millipore).

\section{$178 \quad 2.4$ In vitro fluorescence and bioluminescence quantification in RED parasites}

179 To perform the parasite density / fluorescence-bioluminescence intensity assay with the RED

180 PCF and BSF trypanosomes, as well as with promastigotes of the RED L. major strain, 181 parasites were counted, centrifuged and resuspended in fresh SDM-G, HMI-11 and M199 182 medium, respectively. Then, $100 \mu$ ( or $10^{7}$ parasites) of this suspension were transferred into 183 black clear-bottom 96-well plates and serial 2-fold dilutions were performed in triplicate 184 adjusting the final volume to $200 \mu \mathrm{l}$ with $300 \mu \mathrm{g} / \mathrm{ml}$ of beetle luciferin (Promega, France). 185 Luciferase activity was quantified after 10 minutes of incubation with a microplate reader 186 Infinite $\AA 200$ (Tecan) or an IVIS $₫$ Spectrum imager (Perkin Elmer), following the instructions 187 of the Promega Luciferase Assay System. After removal of intensity values from parasite188 free medium corresponding to the background noise, results were analyzed as mean \pm SD of 189 three independent experiments.

1902.5 Tsetse fly maintenance, infection by RED Trypanosoma brucei brucei and 191 dissection

192 Glossina morsitans morsitans tsetse flies were maintained in Roubaud cages at $24^{\circ} \mathrm{C}$ and $19360 \%$ hygrometry and fed through a silicone membrane with fresh mechanically defibrinated 194 sheep blood. Teneral males (between $24 \mathrm{~h}$ and $72 \mathrm{~h}$ post-emergence) were allowed to ingest 195 parasites in culture medium during their first meal through a silicone membrane. Here, we 196 used either (i) cultured PCF trypanosomes at $5 \times 10^{6}$ cells per millilitre in SDM79 medium 197 supplemented with $10 \%$ foetal bovine serum, $8 \mathrm{mM}$ glycerol and $10 \mathrm{mM}$ glutathione (MacLeod 
et al., 2007), or (ii) freshly in vitro differentiated stumpy BSF trypanosomes resuspended at $2 \times 10^{6}$ cells per millilitre in SDMG medium supplemented with $10 \mathrm{mM}$ glutathione (MacLeod et

200 al., 2007).

201 Flies were starved for at least 24 hours before being dissected. After rapid isolation of the salivary glands in a drop of phosphate buffer saline (PBS), whole tsetse alimentary tracts, from the distal part of the foregut to the malpighian tubules, were dissected and arranged lengthways in a another drop of PBS as previously described (Rotureau et al., 2011; Rotureau et al., 2012). Isolated organs were then either scrutinized under a microscope at high magnification and imaged, or individually dilacerated, dried on the slide and further treated for immuno-fluorescence (Rotureau et al., 2014).

208 Salivation tests were performed one month after the infective meal. First, cold-anesthetized 209 flies were rapidly screened for the presence of a red fluorescent signal through the cuticle of 210 their ventral abdomen under an epifluorescence M165FC stereomicroscope (Leica) at the

211 10x magnification. Positive flies were sorted, starved for 48h, and individual flies were allowed to probe on warm clean glass slides for 5 minutes. Saliva drops were eventually checked for the presence of fluorescent metacyclic trypanosomes under a DMIL LED epifluorescence microscope (Leica) at the x60 magnification.

\subsection{Mouse strains and ethical statements}

216 Wild type BALB/C and C57BL/6J mice from Janvier Laboratory were used as models for 217 experimental infection and monitoring of the bioluminescence with IVIS Spectrum and IVIS 218 CT. In addition, to allow for further 3D intravital imaging of the lymphatic and blood systems, 219 C57BL/6J-Flk1-EGFP mice expressing a GFP-tagged Kdr (Flk1) gene encoding the vascular 220 endothelial growth factor receptor 2 (VEGFR-2), were also used (Ema et al., 2006). This 221 study was conducted in strict accordance with the recommendations from the Guide for the 222 Care and Use of Laboratory Animals of the European Union (European Directive 223 2010/63/UE) and the French Government. The protocol was approved by the "Comite 224 d'éthique en expérimentation animale de l'Institut Pasteur" CETEA 89 (Permit number: 20122250043 and 2016-0017) and undertaken in compliance with Institut Pasteur Biosafety 226 Committee (protocol CHSCT 12.131). BR is authorized to perform experiments on vertebrate 227 animals (license \#A-75-2035) and is responsible for all the experiments conducted personally 228 or under his supervision.

\subsection{Mouse infections with RED parasites}

230 RED BSF ( $10^{5}$ parasites/mouse) were used to inoculate seven-week-old male C57BL/6J or 231 BALB/c mice (Janvier, France) by the IP route. Parasitemia was assayed daily by automated 232 fluorescent cell counting with a Muse cytometer (Merck-Millipore, detection limit at $5 \times 10^{2}$ 
233 parasites $/ \mathrm{ml}$ ) according to the manufacturer's recommendations. Alternatively, mice were

234 infected via the natural route, i.e. by infective bites: tsetse flies with a mature salivary gland

235 infection, assessed by a salivation test, were individually allowed to bite on the belly of a

236 naïve mouse for about 15 minutes, and blood absorption was further verified by examination

237 of their abdomen.

238 Seven-week-old male BALB/C mice were injected with $1 \times 10^{6}$ L. major

239 PpyRE9H/TY1/tdTomato stationary-phase promastigotes (4 to 5-day old) in the hind

240 footpads. After 4 weeks, animals were euthanized by cervical dislocation and popliteal lymph

241 nodes were dissected for imaging. The total number of living parasites invading the target

242 organs (popliteal lymph node draining the injected site) was calculated from serially-diluted

243 single-cell suspensions and dispensed into 96-well plates. Parasitic load expressed as

244 Limiting Dilution Assay Units (LDAU) after ten days of differentiation of amastigotes into

245 promastigotes. The number of parasites was calculated as follows: Limit Dilution Assay Units

$246($ LDAU $)=$ (geometric mean of titre from quadruplicate cultures) $x$ (reciprocal fraction of the

247 homogenized organ added to the first well). The titre was the reciprocal of the last dilution in

248 which parasites were observed (Lima et al., 1997).

$249 \quad 2.8$ Ex vivo fluorescence imaging of RED parasites

250 For live fluorescence imaging of Leishmania promastigotes and all trypanosome stages, 251 parasites in blood, in culture medium or isolated from tsetse fly midgut were observed either 252 (i) with a DMI4000 microscope (Leica) and images were acquired with a Retiga-SRV camera 253 (Q-Imaging), or (ii) with a DMIL LED microscope (Leica) and images were acquired with a 254 digital color DFC450 C camera (Leica). Trypanosomes in tsetse organs were directly 255 monitored with a M165FC stereomicroscope (Leica) with a monochromatic DFC30000 G 256 camera (Leica). For intracellular Leishmania amastigote observations, peritoneal 257 macrophages were collected from one BALB/c mouse. Then, $5 \times 10^{5}$ cells were plated on lbidi 258 chambers and infected at a ratio of 10 stationary-phase promastigotes per macrophage as 259 previously described, and maintained for $72 \mathrm{~h}$ at $37^{\circ} \mathrm{C}$ in $5 \% \mathrm{CO}_{2}$ (Spath et al., 2000). 260 Intracellular amastigotes were then observed with a DMI4000 microscope (Leica) and 261 images were acquired with a Retiga-SRV camera (Q-Imaging). For figure preparation, 262 specific ROI were selected and fluorescent frames were normalized to the same scale of 263 intensity with ImageJ (NIH).

\subsection{In vivo fluorescence imaging of RED parasites}

265 RED trypanosomes were monitored in the ear of Kdr (Flk1) C57BL/6J Rj mice by spinning266 disk confocal microscopy as described previously (Capewell et al., 2016). Briefly, mice were 267 first anaesthetised by IP injection of ketamine (Imalgene 1000 at $125 \mathrm{mg} / \mathrm{kg}$ ) and xylazine 
268 (Rompun $2 \%$ at $12.5 \mathrm{mg} / \mathrm{kg}$ ). Mice were wrapped in a heating blanket and placed on an 269 aluminium platform with a central round opening of $21 \mathrm{~mm}$ in diameter. A coverslip was 270 taped on the central hole and the mouse was positioned so that the ear was lying on this 271 oiled coverslip. Imaging was performed using an UltraView ERS spinning-disk confocal 272 system (Perkin Elmer) with a x40 oil objective (1.3 numerical aperture). Movies were 273 acquired by an EM-CCD camera (Hamamatsu) controlled by the Volocity software (Perkin 274 Elmer) with an exposure time of $500 \mathrm{~ms}$ for a total of 30 to $120 \mathrm{~s}$. Images were analysed 275 using ImageJ $1.48 \mathrm{v}$ and its plugin Bio-formats importer $(\mathrm{NIH})$.

\section{$276 \quad 2.10$ In vivo bioluminescence imaging of RED parasites}

277 Infection by bioluminescent parasites was monitored daily by detecting the bioluminescence 278 in whole animals with the IVIS $®$ Spectrum imager (Perkin Elmer). The equipment consists of 279 a cooled charge-coupled camera mounted on a light-tight chamber with a nose cone delivery 280 device to keep the mice anaesthetized during image acquisition with $1.5 \%$ isoflurane. D281 luciferin potassium salt (Promega) stock solution was prepared in PBS at $33.33 \mathrm{mg} / \mathrm{ml}$, filter282 sterilized and stored in a $-20^{\circ} \mathrm{C}$ freezer. To produce bioluminescence, mice were inoculated 283 IP with $150 \mu \mathrm{l}$ of D-luciferin stock solution $(250 \mathrm{mg} / \mathrm{kg})$. After 10 minutes of incubation to allow 284 substrate dissemination, all mice were anaesthetized in an oxygen-rich induction chamber 285 with $2 \%$ isoflurane, and images were acquired by using automatic exposure (30 seconds to 5 286 minutes) depending on signal intensity. Images were analyzed with Living Image software 287 version 4.3.1 (Perkin Elmer). Data were expressed in average radiance $\left(\mathrm{p} / \mathrm{s} / \mathrm{cm}^{2} / \mathrm{sr}\right)$ 288 corresponding to the total flux of bioluminescent signal according to the selected area (total 289 body of the mouse here). The background noise was removed by subtracting the 290 bioluminescent / fluorescent signal of the control mouse from the infected ones for each 291 acquisition.

292 Morphological / anatomical imaging technologies, such as computed tomography (CT) can 293 be used to provide a combination of functional and anatomical data ensuring that maximum 294 information is extracted from the infected animal model (Willmann et al., 2008). Longitudinal 295 imaging is also used in pre-clinical studies to follow the progress of a disease or measure the 296 effect of a therapeutic (Lim et al., 2011). Bioluminescence was acquired with a cooled CCD 297 camera using the IVIS $₫$ Spectrum CT scanner (Perkin Elmer). Each animal was injected with $298150 \mathrm{mg} / \mathrm{kg}$ of D-luciferin IP $10 \mathrm{~min}$ prior to imaging. Six spectrally resolved images were 299 acquired using emission filters at 560, 580, 600,620,640, and $660 \mathrm{~nm}$ with a bandwidth of $30020 \mathrm{~nm}$ each and an exposure time of 180 seconds. 3D tomographic reconstruction and 301 superposition over the CT volume was performed using the DLIT algorithm available in the 302 Living Image software 4.3. (Perkin Elmer), which uses single-view multispectral 303 bioluminescence images to perform the reconstruction with segmentation of the CT images 
304 in order to provide the mouse body boundary. The bioluminescence signal emitted by RED

305 AnTat 1.1E parasites was monitored 4 days after the inoculation of $10^{5}$ BSF by IP route in 306 BALB/c mice.

\section{$307 \quad 2.11$ Immuno-fluorescence analysis and immuno-histology}

308 For immuno-histology, samples from the most bioluminescent skin regions were fixed in $4 \%$ 309 paraformaldehyde over-night, processed into paraffin blocks and trimmed. Sections were 310 processed for immuno-histochemical staining as described above. For immuno-fluorescence, 311 parasites were settled on poly-L-lysine coated slides and fixed in methanol at $-20^{\circ} \mathrm{C}$ for 5 312 seconds and re-hydrated in PBS for 10 minutes. Slides were incubated with the BB2 primary 313 antibody diluted in PBS containing $0.1 \%$ bovine serum albumin for 45 minutes at $37^{\circ} \mathrm{C}$. The 314 BB2 monoclonal antibody targets the Ty1-tag (mouse lgG1, up to 1/10) (Bastin et al., 1996). 315 Slides were then washed and incubated with an anti-mouse-lgG1 secondary antibody 316 coupled to Cy3 (Jackson) for 30 minutes at $37^{\circ} \mathrm{C}$. Slides were finally stained with $4^{\prime}, 6-$ 317 diamidino-2-phenylindole (DAPI) for visualization of kinetoplast and nuclear DNA content, 318 and mounted under cover slips with ProLong antifade reagent (Invitrogen), as previously 319 described (Rotureau et al., 2011). Samples were observed either with a DMR microscope 320 (Leica) and images were captured with a CoolSnap HQ camera (Roper Scientific), or with a 321 DMI4000B microscope (Leica) and images were acquired with an ORCA-03G camera 322 (Hamamatsu). Image acquisition was controlled using Micro-manager (NIH) and images 323 were taken with the $\mathrm{min} / \mathrm{max}$ threshold set at maximum.

\section{$324 \quad 2.12$ Western blotting}

325 For western blotting, samples were prepared by washing cells in serum-free medium before 326 dilution with PBS and boiling in Laemmli for 5 minutes. SDS-PAGE separation was carried 327 out after loading 10,1 and $0.1 \mu \mathrm{g}$ of total proteins per lane (corresponding to $10^{6}, 10^{5}$ and $32810^{4}$ cells, respectively). Proteins were transferred onto polyvinylidene fluoride membranes for 3297 minutes by using the Trans-Blot $^{\circledR}$ Turbo $^{\text {TM }}$ technology (Bio-Rad), and blocked with $5 \%$ 330 skimmed milk in PBS with $0.1 \%$ Tween 20 for $1 \mathrm{~h}$ prior to probing. The following primary 331 antibodies were used by dilution in $2 \%$ milk in PBS with $0.1 \%$ Tween 20: L8C4 (non-diluted) 332 (Kohl et al., 1999) and BB2 (1:50) (Bastin et al., 1996). Anti-mouse horseradish peroxidase333 conjugated secondary antibodies (Amersham Biosciences) were used at a 1:20,000 dilution. 334 Membranes were washed in PBS with $0.1 \%$ Tween 20 after blocking, between probing with 335 primary and secondary antibodies and prior to band detection by chemiluminescence (GE 336 healthcare). 


\subsection{Measurements, normalization and statistical analysis}

338 Normalization of signals was carried out by parallel manipulation of $\mathrm{min} / \mathrm{max}$ signals in 339 Image (NIH). For clarity purposes, brightness and contrast of several pictures were adjusted 340 after their analysis in accordance with editorial policies. Statistical analyses and plots were 341 performed with XLSTAT 2015.4.01 (Addinsoft) and Excel 2011 (Microsoft), respectively. 342 Statistical analyses include linear regression analyses for bioluminescence / fluorescence 343 intensity Vs. parasite density, ANOVA for growth curves, and ANCOVA for fly infection rates, 344 with Tukey ad-hoc post-tests for inter-group comparison, all with 95\% confidence. 


\section{Results}

We have generated a new chimeric triple reporter recombinant protein of 1,036 aa named

348 PpyRE9H/TY1/tdTomato and composed by the fusion of (i) the human codon-optimized red349 shifted version of the North American firefly Photinus pyralis luciferase (Branchini et al., 350 2005), (ii) a short amino acid sequence known as Ty1-tag (Bastin et al., 1996), and (iii) the 351 tdTomato fluorescent protein (Fig. S1).

352 During the plasmid-construct amplification in E. coli DH5a, the fusion protein expression was 353 attested by the red colour of bacterial pellets and further confirmed under an epifluorescence 354 microscope, demonstrating the potential usefulness of this construct also in prokaryotic cells.

355 The triple reporter construct was then expressed in the cytoplasm of the eukaryotic protist 356 parasites Leishmania major and Trypanosoma brucei brucei. These strains will be further 357 referred to as the RED parasites in the entire article.

\subsection{In vitro and in vivo applications with RED L. major parasites}

359 In order to explore the usefulness of the novel triple reporter construct, a transgenic 360 Leishmania major strain expressing the PpyRE9H/TY1/tdTomato was generated. 361 Promastigotes of $L$. major Friedlin reference strain were electroporated and selected in semi362 solid plates to obtain single fluorescent and bioluminescent clones. The presence of the 363 construct was checked by PCR on genomic DNA (Fig. S2A-B). Parasites were scrutinized 364 under an epifluorescence microscope and their fluorescence was compared to that of wild 365 type cells (WT) (Fig. 1A-B). A strong and specific red fluorescent signal was homogenously 366 distributed in both the cytoplasm and the flagellum of RED promastigote cells only, but it was 367 absent from their nucleus. The fluorescence of RED cells was further confirmed at the 368 population level by flow cytometry analysis (Fig. 1C-D). Moreover, parasite proliferation in 369 vitro was comparable to that of the parental cell line (Fig. 1E).

370 A linear correlation was found between the number of cells and the emission of 371 bioluminescence, by measuring the signal in vitro in 96-well plates with an IVIS Spectrum 372 imager (Fig. 2A), being able to detect as few as $10^{3}$ parasites (Fig. 2B). In parallel, this linear 373 correlation was verified in a TECAN multiwell-plate reader for 5 RED sub-clones (Fig. 2C). 374 Similarly, a linear correlation between the fluorescent signal intensity and the number of 375 parasites was observed with both the imager (Fig. 2D), with a limit of detection at $10^{4}$ cells 376 (Fig. 2E), and the plate reader for 5 RED sub-clones (Fig. 2F).

377 To test the efficiency of the RED L. major in vivo, the virulence of the transfected parasites 378 was firstly recovered by passing the strain once through BALB/c mice. In order to determine 379 whether RED L. major parasites could be detected in vivo using whole-body imaging, $10^{6}$ 380 stationary-phase parasites were injected subcutaneously into the hind footpads of BALB/C 
mice. Lesion progression was monitored both by the direct measurement of the 382 bioluminescence (Fig. 3A) and fluorescence (Fig. 3B) emitted by RED amastigotes recorded 383 in the IVIS Spectrum, and by the follow-up of the hind-limb lesions over four weeks (Fig. 3C384 D). The bioluminescent signal start to increase from 5 days post-infection up to the end of the experiment, reaching a maximum radiance of $6.3 \times 10^{6} \mathrm{p} / \mathrm{s} / \mathrm{cm}^{2} / \mathrm{sr}$ after four weeks of infection. However, in vivo follow-up of the fluorescence during the infection course showed that the fluorescent signal was visible only from the $3^{\text {rd }}$ week after the infection, leading to the expected conclusion that bioluminescence was much more sensitive to evaluate the progression of infection in this context (Fig. 3C-D). After 29 days, mice were sacrificed and the popliteal lymph nodes were extracted and imaged (Fig. 3E-F). Parasitic load (LDAU) in the popliteal lymph node draining the lesion of infected animals, determined by the limiting dilution method, was $4.8 \times 10^{6}$ as an average of both infected organs per mouse. These results are in accordance with regular parasitic load experiments.

394 Then, in vitro infection was performed in freshly collected resident peritoneal macrophages in order to allow parasites to differentiate into intracellular amastigotes forms in which the expression of the triple marker was confirmed as assessed by the presence of a specific red fluorescent signal (Fig. S3A).

\subsection{In vitro uses of RED T. brucei parasites}

In order to explore the usefulness of the novel triple reporter construct in another 400 trypanosomatid parasite, transgenic Trypanosoma brucei brucei mammalian bloodstream 401 forms (BSF) and insect procyclic forms (PCF) were transformed with the pTSARib402 PpyRE9H-TY1-tdTomato vector and immediately sub-cloned by limiting dilution. The 403 presence of the construct was checked by PCR on genomic DNA (Fig. S2). RED PCF 404 trypanosomes in culture were scrutinized under a fluorescence microscope for a direct visual confirmation of their fluorescent phenotype as compared to WT cells. As expected, the triple reporter was homogenously distributed in the cytoplasm, and in a lesser extend in the flagellum, only in transformed parasites, but it was absent from their nucleus (Fig. 4A-B). The

408 fluorescence of RED cells was further confirmed at the population level by flow cytometry 409 analysis (Fig. 4C-D). Similar observations were done with RED BSF trypanosomes, yet with 410 a lower overall fluorescence intensity as compared to that in RED PCF cells (Fig. 4E-H). 411 Although the proliferation of PCF was significantly slower in transformed parasites compared 412 to WT cells (doubling time of $14.5 \mathrm{~h}$ and $10.5 \mathrm{~h}$, respectively, ANOVA p $=0.034$ ) (Fig. $4 \mathrm{I}$ ), the 413 BSF proliferation rate in vitro was comparable to that of the parental cell line (doubling time 414 of $6.5 \mathrm{~h}$ for both) (Fig. $4 \mathrm{~J}$ ).

415 First, in order to verify the reliability of the bioluminescent marker as well as to define its 416 detection threshold, a parasite density / bioluminescence intensity analysis was performed in 
96-micro-well plates with an IVIS Spectrum imager (Fig. 5A-B). Parasite density and

418 bioluminescence intensity were correlated when bioluminescence levels were higher than

$41910^{4} \mathrm{p} / \mathrm{s} / \mathrm{cm}^{2} / \mathrm{sr}$, corresponding to about $10^{2}$ RED BSF or RED PCF parasites, allowing 420 estimation of the parasite density from in vivo imaging over this threshold (Fig. $5 \mathrm{C}$ ). In 421 parallel, this linear correlation was verified in a TECAN multiwell-plate reader for several 422 RED sub-clones of both parasite stages (Fig. 5D-E), yet with some bioluminescence intensity 423 level variations between clones, especially for RED PCF. Second, the fluorescent signal 424 emitted by these RED $T$. brucei parasites was measured in a IVIS fluorescence reader and 425 appeared to be correlated to their number in a dynamic range starting from $10^{3} \mathrm{PCF}$ and $10^{4}$ 426 BSF (Fig. 5F-G), although the overall fluorescence intensity level in RED BSF was much 427 lower compared to that in RED PCF (Fig. 5H). This is possibly due to the existence of BSF428 specific chemical conditions in the cytosol, such as $\mathrm{pH}$ variations and / or high localized 429 concentrations of cations, that may affect tdTomato activity, or to a higher photobleaching 430 rate at $37^{\circ} \mathrm{C}$, rather than to a lower turn-over or to a reduced folding efficiency, since the 431 luciferase activity was not affected, as assessed by high bioluminescence levels. This 432 observation was further confirmed by testing several subclones of both stages in a plate 433 reader (Fig. 5l-J).

\subsection{In vivo uses of RED T. brucei parasites}

435 To test whether RED trypanosomes could be monitored in vivo in their insect vector, 436 Glossina morsitans morsitans teneral males were experimentally infected with RED PCF 437 parasites through a silicone membrane. One month after the infective meal, flies were 438 screened under an epifluorescence binocular microscope for the presence of a specific red 439 fluorescence through their abdominal cuticle, reflecting the presence of RED parasites in 440 their midgut (Fig. 6A-B). To assess the stability of the triple reporter expression during the 441 course of the infectious process, i.e. without any selective antibiotic pressure, both negative 442 and positive flies were dissected and the presence of parasites in their midgut was verified 443 by both light and epifluorescence microscope examination at a higher magnification (Fig. 6C444 E). Living parasites were only detected in flies that were initially scored positive by 445 fluorescence examination of the intact abdomen, demonstrating the stability of the triple 446 reporter in vivo (Fig. $6 \mathrm{~F}$ and Movie1). It is noteworthy that the red fluorescent component of 447 the triple reporter could also be used to directly scrutinize the parasite development, motility 448 and interactions in vivo or ex vivo within the tsetse host tissues and organs (Fig. 6E-F and 449 Movie1) (Schuster et al., 2017). Then, in order to assess the virulence of RED parasites in 450 the tsetse fly, a total of 17 batches of flies were fed with either WT BSF, WT PCF, RED BSF 451 or RED PCF parasites in culture. Experimental infections were performed over a one-year 452 period by comparing strains at least two by two. In total, 606 flies were dissected after one 
month, and the infection rates in the midgut and salivary glands were compared between

454 groups (Fig. 6G). For WT parasites, fly infection by BSF gave rise to significantly higher 455 infection rates, especially in the midgut $(p=0.019$, ANOVA's Tukey ad-hoc post-test, 95\% 456 confidence). In total, RED parasites produced similar infection rates as compared to WT 457 controls, except for the RED BSF that were significantly less prone than WT BSF to produce 458 mature infection in the salivary glands $(p=0.003$, ANOVA's Tukey ad-hoc post-test, $95 \%$ 459 confidence).

460 Conveniently, the possibility of assessing the infection by a simple observation of living flies 461 allowed us to anticipate the number of flies which could be potentially infected at the salivary 462 gland level. Therefore, in another experiment, only fluorescent flies were used for salivation 463 tests one month after the infective meal in order to sort living tsetse flies with a mature 464 salivary gland infection for further natural transmission experiments. Single flies with salivary 465 gland infection were then used to naturally infect mice, and the course of the infection was 466 monitored daily by acquiring the bioluminescent signal emitted by the parasites in the entire 467 organism with an IVIS Spectrum imager (Fig. 7A). Mice bitten by uninfected flies and not 468 bitten at all were used as controls. The parasitemia was also daily quantified by detecting 469 RED fluorescent BSF in $5 \mu$ l blood samples with a Muse bench flow-cytometer. In parallel, 470 the parasitemia was verified by manual counting of living parasites with a KOVA 471 hemocytometer (Fig. 7B). Similar parasitemia quantifications were obtained by both methods 472 (not shown), demonstrating that the fluorescence, although being limited in RED BSF as 473 compared to RED PCF, was stably maintained over the course of the infection. A significant 474 bioluminescent signal was detected as soon as after 1 to 2 days and was persisting until the 475 end of the experiment, whereas the parasitemia was only first detected after 17 days and 476 further fluctuated by characteristic waves (Fig. 7C). This demonstrates that a substantial 477 population of parasites remained extravascular during the entire course of the infection. As 478 assessed by ventral and dorsal bioluminescence imaging, both the parasite distribution and 479 density evolved in space and time during the entire infection course (Fig. 7C). This 480 experiment reproduces our previous study where the bioluminescent signal intensity was 481 used for quantifying the correlated parasite densities (Capewell et al., 2016). In the latter 482 study, mice infected with RED BSF were sacrificed and dissected to record the 483 bioluminescent signal in isolated organs: in contrast to the bioluminescent signals detected in 484 the spleen, the liver, the inguinal and cervical lymph nodes and some adipose tissues, the 485 skin was found to constitute the major anatomical reservoir of parasites even in the absence 486 of detectable parasites in the blood (Capewell et al., 2016). Alternatively, in order to study the 487 differential anatomical distribution of the RED parasites in living mice, we attempted to test 488 longitudinal imaging with the IVIS Spectrum CT imager. The computed tomography 489 application allowed us to extract the spatial 3D-localization of RED parasites, although only 
490 in heavily infected organs (spleen, liver and lymph nodes) (Fig. 7D). To refine the

491 observation of living parasites developing in the skin at the cell level, the same naturally-

492 transmitted RED trypanosomes were also imaged in vivo using a spinning-disk confocal

493 microscopy setting in the C57BL/6J-Flk1-EGFP mouse line that present green fluorescent

494 endothelial cells in the lymphatic and blood vessels (Ema et al., 2006). Strikingly,

495 extravascular trypanosomes were observed gliding and dividing in the dermal layer of the ear

496 (Fig. S3B) (Capewell et al., 2016).

497 In total, the RED trypanosomes were comparable to the parental WT strain in terms of 498 pleomorphism and tsetse infectivity. Both red markers were stably expressed in all stages of 499 the parasite cycle, both in vivo and in vitro, in cells kept from $27^{\circ} \mathrm{C}$ to $37^{\circ} \mathrm{C}$, in the absence of 500 any antibiotic pressure, and over at least 3 months in total. All these features allowed us to 501 scrutinize the kinetics of the same infection at different scales in vivo (single cells, tissue 502 level and whole body).

\subsection{Immuno-detection of the triple reporter construct with the TY1 tag}

504 The bioluminescent and fluorescent properties of the two reporter proteins used in the construct mainly rely on their conformation and on the physico-chemical nature of the microenvironment. For this reason, common regular protocols, such as for electron microscopy, histology or protein biochemistry are known to at least partially alter protein structures, which results in a loss of enzymatic activity, fluorescence emission or immune-specificity. For these reasons, we have introduced the small Ty1-tag between the red-shifted luciferase and the tdTomato in order to stabilize the fusion protein and to serve as a third reporter in specific conditions where the two other reporters would not be detected. The Ty1-tag can be recognized by two different monoclonal antibodies with overlapping binding sites: BB2, which is an immunoglobulin G (lgG1) and TYG5, which is an immunoglobulin A.

514 The expression and accessibility of the Ty1 epitope was first assessed by 515 immunofluorescence staining with BB2 in both RED T. brucei PCF (Fig. 8A) and RED $L$. 516 major promastigotes (Fig. 8B) after a 5-seconds methanol fixation. The homogenous 517 distribution of the signal within the entire cytoplasm and flagellum was similar to that obtained 518 directly by recording the tdTomato emission in living cells (Fig.1B and 4B). Then, we 519 performed a western blot in denaturing conditions in order to test the ability of BB2 to detect 520 the Ty1 reporter in total protein samples from RED T. brucei PCF and RED L. major 521 promastigote parasites (Fig. 8C). Different amounts of parasites $\left(10^{6}, 10^{5}\right.$ and $\left.10^{4}\right)$ were 522 tested in order to confirm the correlation between the total amount of proteins and the 523 detection of the tag. A specific band of $110 \mathrm{kDa}$ was revealed only in the protein samples 524 from $10^{5}$ and $10^{6}$ transformed parasites. Finally, highly bioluminescent regions of the skin of 525 naturally infected mice (Fig. 7A) were sampled, fixed in paraformaldehyde, paraffin- 
526 embedded and treated for immuno-histological analysis with BB2. Although no direct red

527 fluorescence was detected, probably as the result of the sample heating for paraffin528 embedment, the BB2 antibody was able to in situ label skin-dwelling-parasites expressing 529 the Ty1 tag (Fig. 8D). These results confirm the potential use of the Ty1-tag included in the 530 chimeric triple reporter fusion protein in a wide variety of immunological assays pre-requiring 531 chemical denaturation and / or heating of the samples.

\section{Discussion and conclusion}

\section{$533 \quad 4.1$ Importance of intravital imaging in research on trypanosomatids}

534 Intravital imaging approaches already led to important discoveries on the biology of 535 trypanosomatid parasites. The use of bioluminescent T. brucei parasites has successively 536 enable different research groups to identify specific anatomical niches that strongly support 537 the preponderance of extravascular parasite populations during the infectious process. Claes 538 and co-workers showed that parasites located in the testis were not cleared by trypanocidal 539 drugs, as they were protected by the blood-testis barrier (Claes et al., 2009). Trindade et al. 540 and Caljon et al. further observed a close association of $T$. b. brucei with adipocytes in fat 541 tissues and subcutaneous adipose layers, respectively (Caljon et al., 2016; Trindade et al., 542 2016). At the same time, together with colleagues from Glasgow, Conakry and Kinshasa, we 543 have demonstrated that the majority of $T$. b. gambiense parasites were actually rather 544 developing in the extravascular matrix of the dermis of murine models, where they were seen 545 actively swimming and dividing (Capewell et al., 2016). In another study by D'Archivio and 546 co-workers, the importance of the late brain invasion by T. vivax parasites was assessed in a 547 dynamic manner by intravital imaging of a bioluminescent strain (D'Archivio et al., 2013). 548 Recently, the principal clinical, immunological and parasitological features associated with 549 visceral leishmaniasis were characterized by in vivo imaging approaches of $L$. donovani 550 transgenic parasites in a golden hamster model (Rouault et al., 2017) and the occurrence of 551 multiple phases of brain invasion and inflammation was further unambiguously demonstrated 552 (Melo et al., 2017). Bioluminescence has also been recently used to monitor the evolution of 553 the T. cruzi parasite load in the digestive tract of its triatomine vector Rhodnius prolixus (Dias 554 Fde et al., 2015).

555 Applied to drug development in high-throughput (HTS) in vitro assays as well as in in vivo 556 murine models, bioluminescent and fluorescent trypanosomatid reporter strains have also 557 proved their usefulness. Intravital multi-photon microscopy revealed that diminazene was 558 unable to target $T$. b. brucei parasites that have already crossed the blood-brain barrier three 559 weeks after the infection in a mouse model of stage 2 sleeping sickness (Myburgh et al., 560 2013). Reimão and co-workers evaluated the efficacy of miltefosine in vivo in a luciferase- 
expressing $L$. infantum chagasi experimental model of infection in hamsters that mimics

562 human disease (Reimao et al., 2015). A non-invasive model of lesion suppression has been

563 recently validated for the semi-HTS screening of compounds against cutaneous

564 leishmaniasis by using a bioluminescent strain of $L$. major (Caridha et al., 2017). Other

565 studies have taken advantage of the use of fluorescent and / or bioluminescent parasites to

566 screen myriads of compounds in multi-well plates. Annang and co-workers validated a HTS

567 platform to screen a subset of microbial extracts against the 3 trypanosomatid-borne 568 diseases in 384-well plates by using, among them, bioluminescent-L. donovani parasites 569 (Annang et al., 2015). The use of ex vivo cultures from infected tissues or organs has been 570 explored and exploited as well by some other research groups with bioluminescent $L$. 571 donovani (Osorio et al., 2011) and L. major (Peniche et al., 2014), as well as with infrared572 emitting L. infantum parasites (Calvo-Alvarez et al., 2015b).

\subsection{Novel perspectives offered by the triple reporter}

574 Most of these studies are relying on the use of constructs encoding for single green 575 fluorescent probes or single luciferases emitting in the green. This is not optimal for intravital 576 imaging due to the high absorption of the light by the tissue components at these 577 wavelengths, and this also limits the monitoring to a single scale, i.e. whole organism or 578 tissue / cell level. To bypass this technical bias, we have simultaneously used two reporters 579 stably producing light in the red wavelengths with a reduced absorption by tissues, resulted 580 in an enhanced sensitivity of the monitoring, with equivalent detection thresholds compared 581 to previous studies (Burrell-Saward et al., 2015; McLatchie et al., 2013; Taheri et al., 2015; 582 Van Reet et al., 2014). In the present study, as in the one of McLatchie and colleagues 583 (McLatchie et al., 2013), we used a human codon optimized PpyRE9h sequence that was 584 adapted to T. brucei. However, although this would theoretically have not been optimal for 585 Leishmania, whose genome are more GC-rich, our results demonstrate that this actually 586 doesn't seem to affect the triple marker expression. Taheri and collaborators have monitored 587 and tracked a cutaneous Leishmania infection in real time, by generating stably-transgenic $L$. 588 major parasites that expressed a bi-reporter protein constituted by an EGFP fused to a firefly 589 luciferase. They demonstrated that the simultaneous use of two reporters increases the 590 experimental sensitivity for detection and quantification of parasites in vitro as well as during 591 real-time infection in mice (Taheri et al., 2015). In the present work, the constitutive cytosolic 592 expression of the red triple reporter in African trypanosome and Leishmania strains in mice 593 allowed us to study the kinetics of the infection in the same animal and at different scales by 594 successively using a combination of complementary imaging technologies. We have 595 demonstrated the correct expression of the transgenic constructs with a clear correlation 596 between the number of parasites and the bioluminescent / fluorescent signals in vitro, thus 
permitting the estimation of the parasite load by means of signal acquisition. However, using this methodology for an exact in vivo quantification of the parasite densities in experimental host tissues will further require a higher degree of standardization and some more controls in order to obtain reliable and reproducible quantitative results. In total, the use of this triple reporter led us to access both qualitative and quantitative data on the development of RED $L$. major in a mouse model, as well as on the full parasite cycle of RED T. brucei in the tsetse digestive tract and in the mammalian host after natural transmission. Furthermore, the Ty1tag was also used to refine the analysis of the same biological material after intravital imaging, by immuno-based approaches (western blot, immunofluorescence analyses, immunohistochemistry).

607 It was the powerful combination of both fluorescence and bioluminescence imaging that 608 allowed us to recently formally prove that skin-dwelling parasites were readily ingested by 609 tsetse flies to fulfil their parasite cycle (Capewell et al., 2016). In the context of other HAT 610 research projects, the optimised triple reporter line described here would also possibly 611 enhance the application of in vivo imaging to study stage II African trypanosomiasis in murine 612 models. Indeed, it will provide a new framework for investigating host-parasite relationships, 613 particularly in the context of CNS infections. In addition, it should be ideally suited to drug 614 evaluation programmes. This RED triple reporter construct represents a powerful tool that 615 could also be useful in many other fields of research where cellular model can be 616 transformed. According to the plasmid vector used and to its way of integration, the signal 617 intensities could theoretically be modulated. Moreover, although the size of the triple reporter 618 is not exactly appropriated, one could think about its potential use for protein tagging. 619 Possible simultaneous qualitative and quantitative applications in research are the use of its 620 bioluminescence component for in vitro HTS drugs screening and in vivo monitoring 621 (microbiology, immunology, parasitology, oncology, pharmacology, vaccinology...), the use 622 of its fluorescence for in vitro assays (HTS drug screening, investigation of biological 623 processes...) and in vivo monitoring (microbiology, immunology, parasitology, oncology...), 624 and the use of the Ty1-tag for immuno-detection in fixed and treated samples after real-time 625 intravital imaging.

626 


\section{Conflict of interest statement}

628 The authors declare that the research was conducted in the absence of any commercial or

629 financial relationships that could be construed as a potential conflict of interest.

\section{Author contribution statement}

631 ECA, CCT and AC contributed to the experiments.

632 ECA and BR contributed to study design, data analysis and manuscript writing.

\section{Funding statement}

634 This work was funded by the Institut Pasteur (PTR-403), by a French Government 635 Investissement d'Avenir programme, Laboratoire d'Excellence "Integrative Biology of 636 Emerging Infectious Diseases" (ANR-10-LABX-62-IBEID) and by the French National

637 Agency for Scientific Research (Young Researcher Grant ANR-14-CE14-0019-01). ECA and

638 CCT were funded by a French Government Investissement d'Avenir programme, Laboratoire 639 d'Excellence "Integrative Biology of Emerging Infectious Diseases" (ANR-10-LABX-62-IBEID) 640 and by the French National Agency for Scientific Research (Young Researcher Grant ANR641 14-CE14-0019-01). AC and BR were funded by the Institut Pasteur.

\section{Acknowledgements}

643 We acknowledge Pascal Pescher and Gerald Späth, Keith Gull, Martin Taylor and Bruce 644 Branchini for providing various reagents. We are grateful to Philippe Bastin for his strong 645 human and scientific support. We especially thank Marie-Anne Nicola and Jean-Yves 646 Tinevez from the Dynamic Imaging Platform, Imagopole, CiTech, at the Institut Pasteur, for 647 their very efficient support in intravital imaging. 
649 Fig. 1: Fluorescence pattern and growth of the RED L. major.

650 (A-B) Live epifluorescence microscopy images of a WT L. major promastigote (A) and a RED 651 L. major promastigote (B) (normalized TdTomato fluorescence in red and DNA staining in 652 blue). (C-D) Ungated flow-cytometry analysis profiles confirming the non-fluorescence and red fluorescence (in arbitrary units) of WT (C) and RED (D) L. major promastigote populations, respectively. (E) Growth profiles (mean \pm SD from triplicates) of RED L. major promastigotes (in red) compared to their parental WT strain (in blue).

\section{Fig. 2: Quantification of the RED L. major bioluminescence and fluorescence.}

657 Images of the bioluminescent (A) and fluorescent (D) signals emitted by serial 1/2 dilutions of 658 RED L. major promastigotes observed with an IVIS Spectrum in a 96-well plate. The same 659 analysis was performed in triplicates for the RED strain (lines RED 1 to RED3). An equivalent 660 serial dilution of WT promastigotes was used as control (line WT). The last three wells 661 containing only medium were used as blank (red square). Radiance intensity scales are 662 provided in $\mathrm{p} / \mathrm{s} / \mathrm{cm}^{2} / \mathrm{sr}$ on the right of each picture. $(B, E)$ The mean bioluminescent $(B)$ and 663 fluorescent $(E)$ signals $( \pm S D$ ) emitted by RED promastigotes show a significant linear 664 correlation with the number of cells (linear regression analysis; $r^{2}=1.0$ with $p<0.0001$ for $B L$; $665 r^{2}=0.99$ with $p<0.0001$ for fluorescence). (C, F) Bioluminescent (C) and fluorescent $(F)$ 666 signals $( \pm S D$ ) emitted by 5 different RED L. major clones were measured in triplicates with a 667 TECAN plate reader and plotted in arbitrary units (AU) according to the parasite density. A 668 similar linear correlation was observed for all sub-clones.

669 Fig. 3: In vivo imaging applications with the RED L. major.

670 (A-B) In vivo whole-body images of BALB/c mice, including 1 uninfected control (C) and 4 671 infected mice (1 to 4), acquired with an IVIS Spectrum imager. Bioluminescent (A) and 672 fluorescent (B) signals acquired on the hind footpads 28 days post-infection. Radiance 673 intensity scales are provided in $\mathrm{p} / \mathrm{s} / \mathrm{cm}^{2} / \mathrm{sr}$ under each picture. (C, D) Bioluminescent (C) and 674 fluorescent (D) signals on the hind footpads were measured and plotted over the course of 675 the infection. (E, F) RED L. major amastigotes residing in the popliteal draining lymph nodes 676 of 2 mice dissected 4 weeks after inoculation emit bioluminescence $(E)$ and fluorescence $(F)$. 677 Radiance intensity scales are provided in $\mathrm{p} / \mathrm{s} / \mathrm{cm}^{2} / \mathrm{sr}$ under each picture.

678 Fig. 4: Fluorescence pattern and growth of the RED T. brucei PCF and BSF stages.

679 (A-B and E-F) Live epifluorescence microscopy images of WT (A and E) and RED (B and F) 680 T. brucei PCF (A-B) and BSF (E-F) (normalized TdTomato fluorescence in red and DNA 681 staining in blue). (C-D and G-H) Ungated flow-cytometry analysis profiles confirming the non- 
682 fluorescence and red fluorescence (in arbitrary unit) of WT ( $C$ and $G$ ) and RED (D and H) $T$. 683 brucei PCF (C-D) and BSF (G-H) populations, respectively. (I-J) Growth profiles (mean \pm SD

684 from triplicates) of WT (in blue) and RED (in red) T. brucei PCF (I) and BSF (J).

685 Fig. 5: Bioluminescence and fluorescence quantification in the RED T. brucei PCF and 686 BSF stages.

687 Images of the bioluminescent (A-B) and fluorescent (F-G) signals emitted by serial $1 / 2$ 688 dilutions of RED T. b. brucei PCF (A and F) and BSF (B and G) observed with an IVIS 689 Spectrum in 96-well plates. The same analyses were performed in triplicates for the RED 690 strains (lines RED 1 to RED3). An equivalent serial dilution of WT parasites was used as 691 control (lines WT). The last three wells containing only medium were used as blank (red 692 square). Radiance intensity scales are provided in $\mathrm{p} / \mathrm{s} / \mathrm{cm}^{2} / \mathrm{sr}$ on the right of each picture. (C 693 and $H$ ) The mean bioluminescent $(C)$ and fluorescent $(H)$ signals ( \pm SD) emitted by RED 694 PCF (in blue, linear regression analysis with $p<0.0001, r^{2}=0.97$ for $B L$ and $r^{2}=0.96$ for 695 fluorescence) and RED BSF (in red, linear regression analysis with $p<0.0001, r^{2}=0.87$ for $B L$ 696 and $r^{2}=0.97$ for fluorescence) show a significant linear correlation with the number of cells. 697 (D-E and I-J) Bioluminescent (D-E) and fluorescent (I-J) signals ( \pm SD) emitted by 3 different 698 RED T. b. brucei PCF sub-clones ( $D$ and $\mathrm{I}$ ) and 4 different RED $T$. b. brucei BSF subclones 699 (E and J) were measured in triplicates with a TECAN plate reader and plotted in arbitrary 700 units (AU) according to the parasite density. A similar linear correlation was observed for all 701 sub-clones.

702 Fig. 6: Imaging applications with the RED T. brucei in the tsetse fly host.

703 Glossina morsitans morsitans teneral males were fed with RED PCF and observed under an epifluorescence binocular microscope after one month in order to sort infected flies. A strong red fluorescent signal was observed through the ventral abdomen of infected flies (B) but not in non-infected flies (A). This was confirmed by dissection and observation of the fly midgut ( $C$ and $D$, respectively). Fluorescence was even detected dorsally in strongly infected flies

708 (E), and individual RED parasites could be distinguished under the epifluorescence binocular 709 microscope in and outside dissected midguts ( $F$, still image from movie $S 1)$. (G) The virulence of RED parasites was assessed by feeding batches of tsetse flies with either WT

711 BSF, WT PCF, RED BSF or RED PCF parasites resuspended in SDMG culture medium. The 712 histogram shows the infection rates in the midgut and salivary glands observed one month 713 post infection ( ${ }^{*}$ statistically significant difference between indicated groups with $p<0.02$ by ANCOVA's Tukey ad-hoc post-test at 95\% confidence). 

natural transmission.

(A) In vivo whole-body images of C57BL/6J mice acquired with an IVIS Spectrum imager 8, 15 and 22 days after a tsetse fly bite, including one mouse bitten an infected tsetse fly $(+)$, one bitten by a non-infected fly (-) and one non-bitten (0). Radiance intensity scales are provided in $\mathrm{p} / \mathrm{s} / \mathrm{cm}^{2} / \mathrm{sr}$. (B) Live RED BSF in a blood sample from mouse + (DNA in blue). (C)

721 Total ventral (blue squares) and dorsal (green triangles) bioluminescent signals (total 722 radiance in $\mathrm{p} / \mathrm{s} / \mathrm{cm}^{2} / \mathrm{sr}$ ) were measured, normalized to negative controls and plotted over the 723 course of the infection together with the parasitemia (red dots) for mouse +. (D)

724 Bioluminescence and anatomical 3D computed tomography images acquired by an IVIS 725 Spectrum CT on a BALB/c mouse 29 days post-inoculation. The 3D reconstruction view on 726 the right panel has been decomposed in pictures along 3 distinct plans showed on the left 727 (coronal in red, sagittal in blue and transaxial in green).

Fig. 8: Detection of RED trypanosomatids with the Ty1-tag after sample alteration by 729 specific treatments.

730 (A, B) Immunofluorescence images of methanol-fixed RED T. brucei PCF (A) and RED $L$. 731 major promastigotes (B) stained with the BB2 antibody (Ty1 in red) and DAPI (DNA in blue). 732 (C) Reactivity of the BB2 monoclonal antibody against the Ty1-tag epitope (110 kDa) by 733 Western Blot analysis. The L8C4 anti-PFR2 antibody (69 kDa) was used as a loading 734 control. Total protein extracts from $10^{6}, 10^{5}$ and $10^{4}$ cells were used to compare the expression levels of the Ty1-tag in RED T. brucei procyclic forms and RED L. major compared to their respective WT control strains. Ladders $(L)$ are provided on both sides of the blot with their respective size in $\mathrm{kDa}$. (D) Immuno-histological analysis of a paraffin-

738 embedded bioluminescent skin section from a mouse infected with RED T. brucei, in situ 739 stained with the BB2 antibody (Ty-1 in red) and DAPI (DNA in blue). A skin-dwelling-parasite 740 expressing the Ty1-tag situated in the extracellular matrix of the dermis is indicated with a 741 white arrow. 


\section{Supplementary material}

743 Fig. S1: The PpyRE9/TY1/tdTomato fusion protein.

744 (A) Schematic view of the protein regions (1,036 aa). (B) DNA sequence (3,111 bp).

745 Fig. S2: Detection of the PpyRE9H/TY1/tdTomato-encoding gene and integration 746 strategies into genomic RRNA loci of T. brucei and L. major.

747 (A) PCR analysis for confirmation of the genomic presence of the triple reporter sequence 748 (primers 1/2) and the specific antibiotic cassettes in RED T.brucei (primers 3/4) and RED $L$. 749 major (primers 5/6) parasites. (B) The upper scheme shows the final structure of the $18 \mathrm{~S}$ 750 RRNA locus of $T$. brucei with the planned integration of the triple marker sequence. The 751 bottom scheme represents the expected integration of the chimeric $3.1 \mathrm{~Kb}$ multiplex reporter 752 protein PpyRE9H/TY1/tdTomato in the genome of $L$. major. PARP SL: procyclin splice site; 753 bsd: blasticidin resistance cassette; utr1: 5' untranslated region of aprt gene; utr2: $1.4 \mathrm{~kb}$ 754 intergenic region from cam operon; and utr3: 5' UTR of dhfr-ts gene; hyg: hygromycin B 755 resistance cassette. Primers 1 and 2 amplify the $3.1 \mathrm{~Kb}$ region of the PpyRE9H-TY1756 tdTomato sequence (1-GGAGGACGCCAAGAACATCAAG; 757 TTACTTGTACAGCTCGTCCATGC); primers 3 and 4 amplify the blasticidin antibiotic 758 cassette (3-ATGGCCAAGCCTTTGTCTCAAG; 4-TTAGCCCTCCCACACATAACCAG); and 759 primers 5 and 6 were used to amplify the hygromycin resistance cassette (5760 ATGAAAAAGCCTGAACTCACCGC; 6-CTATTCCTTTGCCCTCGGACG).

761 Fig. S3: Further imaging applications with RED parasites to monitor host cell / parasite 762 interactions at the cellular level.

763 (A) RED L. major intracellular amastigotes within BALB/c peritoneal macrophages after $72 \mathrm{~h}$ 764 of incubation (DNA in blue and red fluorescent amastigotes in red). (B) Still-image from a 765 spinning-disk confocal microscope movie showing extravascular RED T. brucei parasites (in 766 red) in the surrounding of a vascular or lymphatic vessel (in green) in the ear dermis of a 767 living C57BL/6J-Flk1-EGFP mouse 21 days after an infective bite.

768 Movie S1: RED T. b. brucei in the posterior midgut of a tsetse fly 7 days after the 769 infective meal.

770 Living RED T. b. brucei procyclic parasites observed with a binocular epifluorescence 771 microscope within the posterior midgut of an infected tsetse fly 7 days after the infectious 772 meal. Red fluorescent parasites can be seen swimming outside the organ. 
Aksoy, S., Buscher, P., Lehane, M., Solano, P., Van Den Abbeele, J., 2017. Human African trypanosomiasis control: Achievements and challenges. PLoS Negl Trop Dis 11, e0005454. Alvar, J., Velez, I.D., Bern, C., Herrero, M., Desjeux, P., Cano, J., Jannin, J., den Boer, M., Team, W.H.O.L.C., 2012. Leishmaniasis worldwide and global estimates of its incidence. PLoS One 7, e35671. Annang, F., Perez-Moreno, G., Garcia-Hernandez, R., Cordon-Obras, C., Martin, J., Tormo, J.R., Rodriguez, L., de Pedro, N., Gomez-Perez, V., Valente, M., Reyes, F., Genilloud, O., Vicente, F., Castanys, S., Ruiz-Perez, L.M., Navarro, M., Gamarro, F., GonzalezPacanowska, D., 2015. High-throughput screening platform for natural product-based drug discovery against 3 neglected tropical diseases: human African trypanosomiasis, leishmaniasis, and Chagas disease. J Biomol Screen 20, 82-91. Barquilla, A., Saldivia, M., Diaz, R., Bart, J.M., Vidal, I., Calvo, E., Hall, M.N., Navarro, M., 2012. Third target of rapamycin complex negatively regulates development of quiescence in Trypanosoma brucei. Proc Natl Acad Sci U S A 109, 14399-14404. Bastin, P., Bagherzadeh, Z., Matthews, K.R., Gull, K., 1996. A novel epitope tag system to study protein targeting and organelle biogenesis in Trypanosoma brucei. Mol Biochem Parasitol 77, 235-239.

Beattie, L., Kaye, P.M., 2011. Leishmania-host interactions: what has imaging taught us? Cell Microbiol 13, 1659-1667.

Branchini, B.R., Southworth, T.L., Khattak, N.F., Michelini, E., Roda, A., 2005. Red- and green-emitting firefly luciferase mutants for bioluminescent reporter applications. Anal Biochem 345, 140-148.

796 Brookman, J.L., Stott, A.J., Cheeseman, P.J., Burns, N.R., Adams, S.E., Kingsman, A.J., 797 Gull, K., 1995. An immunological analysis of Ty1 virus-like particle structure. Virology 207, $798 \quad 59-67$.

799 Brun, R., Schonenberger, 1979. Cultivation and in vitro cloning or procyclic culture forms of

800 Trypanosoma brucei in a semi-defined medium. Short communication. Acta Trop 36, 289801292.

802 Burkard, G., Fragoso, C.M., Roditi, I., 2007. Highly efficient stable transformation of 803 bloodstream forms of Trypanosoma brucei. Mol Biochem Parasitol 153, 220-223.

804 Burrell-Saward, H., Rodgers, J., Bradley, B., Croft, S.L., Ward, T.H., 2015. A sensitive and 805 reproducible in vivo imaging mouse model for evaluation of drugs against late-stage human 806 African trypanosomiasis. The Journal of antimicrobial chemotherapy 70, 510-517.

807 Buscher, P., Cecchi, G., Jamonneau, V., Priotto, G., 2017. Human African trypanosomiasis. 808 Lancet. 
809 Caljon, G., Van Reet, N., De Trez, C., Vermeersch, M., Perez-Morga, D., Van Den Abbeele, 810 J., 2016. The Dermis as a Delivery Site of Trypanosoma brucei for Tsetse Flies. PLoS 811 Pathog 12, e1005744.

812 Calvo-Alvarez, E., Alvarez-Velilla, R., Fernandez-Prada, C., Balana-Fouce, R., Reguera, 813 R.M., 2015a. Trypanosomatids see the light: recent advances in bioimaging research. Drug 814 Discov Today 20, 114-121.

815 Calvo-Alvarez, E., Stamatakis, K., Punzon, C., Alvarez-Velilla, R., Tejeria, A., Escudero816 Martinez, J.M., Perez-Pertejo, Y., Fresno, M., Balana-Fouce, R., Reguera, R.M., $2015 b$. 817 Infrared fluorescent imaging as a potent tool for in vitro, ex vivo and in vivo models of visceral 818 leishmaniasis. PLoS Negl Trop Dis 9, e0003666.

819 Capewell, P., Cren-Travaille, C., Marchesi, F., Johnston, P., Clucas, C., Benson, R.A., 820 Gorman, T.A., Calvo-Alvarez, E., Crouzols, A., Jouvion, G., Jamonneau, V., Weir, W., 821 Stevenson, M.L., O'Neill, K., Cooper, A., Swar, N.K., Bucheton, B., Ngoyi, D.M., Garside, P., 822 Rotureau, B., MacLeod, A., 2016. The skin is a significant but overlooked anatomical 823 reservoir for vector-borne African trypanosomes. eLife 5.

824 Caridha, D., Parriot, S., Hudson, T.H., Lang, T., Ngundam, F., Leed, S., Sena, J., Harris, M., 825 O'Neil, M., Sciotti, R., Read, L., Lecoeur, H., Hickman, M., Grogl, M., 2017. Use of Optical 826 Imaging Technology in the Validation of a New, Rapid, Cost-Effective Drug Screen as Part of 827 a Tiered In Vivo Screening Paradigm for Development of Drugs To Treat Cutaneous 828 Leishmaniasis. Antimicrob Agents Chemother 61.

829 Carneiro, M.B., Hohman, L.S., Egen, J.G., Peters, N.C., 2017. Use of two-photon microscopy 830 to study Leishmania major infection of the skin. Methods.

831 Claes, F., Vodnala, S.K., van Reet, N., Boucher, N., Lunden-Miguel, H., Baltz, T., Goddeeris, 832 B.M., Buscher, P., Rottenberg, M.E., 2009. Bioluminescent imaging of Trypanosoma brucei 833 shows preferential testis dissemination which may hamper drug efficacy in sleeping sickness. 834 PLoS Negl Trop Dis 3, e486.

835 Coles, J.A., Stewart-Hutchinson, P.J., Myburgh, E., Brewer, J.M., 2017. The mouse cortical 836 meninges are the site of immune responses to many different pathogens, and are accessible 837 to intravital imaging. Methods.

838 Coombes, J.L., Robey, E.A., 2010. Dynamic imaging of host-pathogen interactions in vivo. 839 Nat Rev Immunol 10, 353-364.

840 D'Archivio, S., Cosson, A., Medina, M., Lang, T., Minoprio, P., Goyard, S., 2013. Non841 invasive in vivo study of the Trypanosoma vivax infectious process consolidates the brain 842 commitment in late infections. PLoS Negl Trop Dis 7, e1976.

843 Dias Fde, A., Guerra, B., Vieira, L.R., Perdomo, H.D., Gandara, A.C., Amaral, R.J., Vollu, 844 R.E., Gomes, S.A., Lara, F.A., Sorgine, M.H., Medei, E., de Oliveira, P.L., Salmon, D., 2015. 845 Monitoring of the Parasite Load in the Digestive Tract of Rhodnius prolixus by Combined 
846 qPCR Analysis and Imaging Techniques Provides New Insights into the Trypanosome Life

847 Cycle. PLoS Negl Trop Dis 9, e0004186.

848 Dias, J.C., 2015. Evolution of Chagas disease screening programs and control programs:

849 historical perspective. Glob Heart 10, 193-202.

850 Ema, M., Takahashi, S., Rossant, J., 2006. Deletion of the selection cassette, but not cis851 acting elements, in targeted Flk1-lacZ allele reveals Flk1 expression in multipotent 852 mesodermal progenitors. Blood 107, 111-117.

853 Forestier, C.L., 2013. Imaging host-Leishmania interactions: significance in visceral 854 leishmaniasis. Parasite Immunol 35, 256-266.

855 Franco, J.R., Cecchi, G., Priotto, G., Paone, M., Diarra, A., Grout, L., Mattioli, R.C., Argaw, 856 D., 2017. Monitoring the elimination of human African trypanosomiasis: Update to 2014. 857 PLoS Negl Trop Dis 11, e0005585.

858 Gonzalez, U., Pinart, M., Sinclair, D., Firooz, A., Enk, C., Velez, I.D., Esterhuizen, T.M., 859 Tristan, M., Alvar, J., 2015. Vector and reservoir control for preventing leishmaniasis. 860 Cochrane Database Syst Rev, CD008736.

861 Huet, D., Blisnick, T., Perrot, S., Bastin, P., 2014. The GTPase IFT27 is involved in both 862 anterograde and retrograde intraflagellar transport. eLife 3, e02419.

863 Julkowska, D., Bastin, P., 2009. Tools for analyzing intraflagellar transport in trypanosomes. 864 Methods Cell Biol 93, 59-80.

865 Kapler, G.M., Zhang, K., Beverley, S.M., 1990. Nuclease mapping and DNA sequence 866 analysis of transcripts from the dihydrofolate reductase-thymidylate synthase $(R)$ region of 867 Leishmania major. Nucleic Acids Res 18, 6399-6408.

868 Kohl, L., Sherwin, T., Gull, K., 1999. Assembly of the paraflagellar rod and the flagellum 869 attachment zone complex during the Trypanosoma brucei cell cycle. J Eukaryot Microbiol 46, 870 105-109.

871 Kooy, R.F., Hirumi, H., Moloo, S.K., Nantulya, V.M., Dukes, P., Van der Linden, P.M., 872 Duijndam, W.A., Janse, C.J., Overdulve, J.P., 1989. Evidence for diploidy in metacyclic 873 forms of African trypanosomes. Proc Natl Acad Sci U S A 86, 5469-5472.

874 Lang, T., Lecoeur, H., Prina, E., 2009. Imaging Leishmania development in their host cells. 875 Trends Parasitol 25, 464-473.

876 Le Ray, D., Barry, J.D., Easton, C., Vickerman, K., 1977. First tsetse fly transmission of the 877 "AnTat" serodeme of Trypanosoma brucei. Ann Soc Belg Med Trop 57, 369-381.

878 Lewis, M.D., Francisco, A.F., Taylor, M.C., Kelly, J.M., 2015. A new experimental model for 879 assessing drug efficacy against Trypanosoma cruzi infection based on highly sensitive in 880 vivo imaging. J Biomol Screen 20, 36-43.

881 Lim, E., Modi, K., Christensen, A., Meganck, J., Oldfield, S., Zhang, N., 2011. Monitoring 882 tumor metastases and osteolytic lesions with bioluminescence and micro CT imaging. $\mathrm{J}$ Vis 883 Exp. 
884 Lima, H.C., Bleyenberg, J.A., Titus, R.G., 1997. A simple method for quantifying Leishmania 885 in tissues of infected animals. Parasitol Today 13, 80-82.

886 Liu, H., Patel, M.R., Prescher, J.A., Patsialou, A., Qian, D., Lin, J., Wen, S., Chang, Y.F., 887 Bachmann, M.H., Shimono, Y., Dalerba, P., Adorno, M., Lobo, N., Bueno, J., Dirbas, F.M., 888 Goswami, S., Somlo, G., Condeelis, J., Contag, C.H., Gambhir, S.S., Clarke, M.F., 2010. 889 Cancer stem cells from human breast tumors are involved in spontaneous metastases in 890 orthotopic mouse models. Proc Natl Acad Sci U S A 107, 18115-18120.

891 MacGregor, P., Rojas, F., Dean, S., Matthews, K.R., 2013. Stable transformation of 892 pleomorphic bloodstream form Trypanosoma brucei. Mol Biochem Parasitol 190, 60-62.

893 MacLean, L., Myburgh, E., Rodgers, J., Price, H.P., 2013. Imaging African trypanosomes. 894 Parasite Immunol 35, 283-294.

895 MacLeod, E.T., Maudlin, I., Darby, A.C., Welburn, S.C., 2007. Antioxidants promote 896 establishment of trypanosome infections in tsetse. Parasitology 134, 827-831.

897 Matlashewski, G., Arana, B., Kroeger, A., Be-Nazir, A., Mondal, D., Nabi, S.G., Banjara, 898 M.R., Das, M.L., Marasini, B., Das, P., Medley, G., Satoskar, A., Nakhasi, H., Argaw, D., 899 Reeder, J., Olliaro, P., 2014. Research priorities for elimination of visceral leishmaniasis. 900 Lancet Glob Health 2, e683-684.

901 McLatchie, A.P., Burrell-Saward, H., Myburgh, E., Lewis, M.D., Ward, T.H., Mottram, J.C., 902 Croft, S.L., Kelly, J.M., Taylor, M.C., 2013. Highly sensitive in vivo imaging of Trypanosoma 903 brucei expressing "red-shifted" luciferase. PLoS Negl Trop Dis 7, e2571.

904 Melo, G.D., Goyard, S., Fiette, L., Boissonnas, A., Combadiere, C., Machado, G.F., Minoprio, 905 P., Lang, T., 2017. Unveiling Cerebral Leishmaniasis: parasites and brain inflammation in 906 Leishmania donovani infected mice. Sci Rep 7, 8454.

907 Mezzanotte, L., Blankevoort, V., Lowik, C.W., Kaijzel, E.L., 2014. A novel luciferase fusion 908 protein for highly sensitive optical imaging: from single-cell analysis to in vivo whole-body 909 bioluminescence imaging. Analytical and bioanalytical chemistry 406, 5727-5734.

910 Myburgh, E., Coles, J.A., Ritchie, R., Kennedy, P.G., McLatchie, A.P., Rodgers, J., Taylor, 911 M.C., Barrett, M.P., Brewer, J.M., Mottram, J.C., 2013. In vivo imaging of trypanosome-brain 912 interactions and development of a rapid screening test for drugs against CNS stage 913 trypanosomiasis. PLoS Negl Trop Dis 7, e2384.

914 Ooi, C.P., Rotureau, B., Gribaldo, S., Georgikou, C., Julkowska, D., Blisnick, T., Perrot, S., 915 Subota, I., Bastin, P., 2015. The Flagellar Arginine Kinase in Trypanosoma brucei Is 916 Important for Infection in Tsetse Flies. PLoS ONE 10, e0133676.

917 Osorio, Y., Travi, B.L., Renslo, A.R., Peniche, A.G., Melby, P.C., 2011. Identification of small 918 molecule lead compounds for visceral leishmaniasis using a novel ex vivo splenic explant 919 model system. PLoS Negl Trop Dis 5, e962. 
920 Patel, M.R., Chang, Y.F., Chen, I.Y., Bachmann, M.H., Yan, X., Contag, C.H., Gambhir, S.S.,

921 2010. Longitudinal, noninvasive imaging of T-cell effector function and proliferation in living

922 subjects. Cancer Res 70, 10141-10149.

923 Peniche, A.G., Osorio, Y., Renslo, A.R., Frantz, D.E., Melby, P.C., Travi, B.L., 2014.

924 Development of an ex vivo lymph node explant model for identification of novel molecules 925 active against Leishmania major. Antimicrob Agents Chemother 58, 78-87.

926 Perez-Molina, J.A., Molina, I., 2017. Chagas disease. Lancet.

927 Reimao, J.Q., Oliveira, J.C., Trinconi, C.T., Cotrim, P.C., Coelho, A.C., Uliana, S.R., 2015.

928 Generation of luciferase-expressing Leishmania infantum chagasi and assessment of 929 miltefosine efficacy in infected hamsters through bioimaging. PLoS Negl Trop Dis 9, 930 e0003556.

931 Reithinger, R., Dujardin, J.C., Louzir, H., Pirmez, C., Alexander, B., Brooker, S., 2007. 932 Cutaneous leishmaniasis. Lancet Infect Dis 7, 581-596.

933 Rotureau, B., Ooi, C.P., Huet, D., Perrot, S., Bastin, P., 2014. Forward motility is essential for 934 trypanosome infection in the tsetse fly. Cell Microbiol 16, 425-433.

935 Rotureau, B., Subota, I., Bastin, P., 2011. Molecular bases of cytoskeleton plasticity during 936 the Trypanosoma brucei parasite cycle. Cell Microbiol 13, 705-716.

937 Rotureau, B., Subota, I., Buisson, J., Bastin, P., 2012. A new asymmetric division contributes 938 to the continuous production of infective trypanosomes in the tsetse fly. Development 139 , 939 1842-1850.

940 Rotureau, B., Van Den Abbeele, J., 2013. Through the dark continent: African trypanosome 941 development in the tsetse fly. Frontiers in cellular and infection microbiology 3, 53.

942 Rouault, E., Lecoeur, H., Meriem, A.B., Minoprio, P., Goyard, S., Lang, T., 2017. Imaging 943 visceral leishmaniasis in real time with golden hamster model: Monitoring the parasite burden 944 and hamster transcripts to further characterize the immunological responses of the host. 945 Parasitol Int 66, 933-939.

946 Schuster, S., Kruger, T., Subota, I., Thusek, S., Rotureau, B., Beilhack, A., Engstler, M., 947 2017. Developmental adaptations of trypanosome motility to the tsetse fly host environments 948 unravel a multifaceted in vivo microswimmer system. eLife 6.

949 Spath, G.F., Epstein, L., Leader, B., Singer, S.M., Avila, H.A., Turco, S.J., Beverley, S.M., 950 2000. Lipophosphoglycan is a virulence factor distinct from related glycoconjugates in the 951 protozoan parasite Leishmania major. Proc Natl Acad Sci U S A 97, 9258-9263.

952 Stanaway, J.D., Roth, G., 2015. The burden of Chagas disease: estimates and challenges. 953 Glob Heart 10, 139-144.

954 Taheri, T., Saberi Nik, H., Seyed, N., Doustdari, F., Etemadzadeh, M.H., Torkashvand, F., 955 Rafati, S., 2015. Generation of stable L. major(+EGFP-LUC) and simultaneous comparison 956 between EGFP and luciferase sensitivity. Exp Parasitol 150, 44-55. 
957 Takai, A., Nakano, M., Saito, K., Haruno, R., Watanabe, T.M., Ohyanagi, T., Jin, T., Okada,

958 Y., Nagai, T., 2015. Expanded palette of Nano-lanterns for real-time multicolor luminescence

959 imaging. Proc Natl Acad Sci U S A 112, 4352-4356.

960 Trindade, S., Rijo-Ferreira, F., Carvalho, T., Pinto-Neves, D., Guegan, F., Aresta-Branco, F., 961 Bento, F., Young, S.A., Pinto, A., Van Den Abbeele, J., Ribeiro, R.M., Dias, S., Smith, T.K., 962 Figueiredo, L.M., 2016. Trypanosoma brucei Parasites Occupy and Functionally Adapt to the 963 Adipose Tissue in Mice. Cell Host Microbe.

964 Van Reet, N., Van de Vyver, H., Pyana, P.P., Van der Linden, A.M., Buscher, P., 2014. A 965 panel of Trypanosoma brucei strains tagged with blue and red-shifted luciferases for 966 bioluminescent imaging in murine infection models. PLoS Negl Trop Dis 8, e3054.

967 Willmann, J.K., van Bruggen, N., Dinkelborg, L.M., Gambhir, S.S., 2008. Molecular imaging 968 in drug development. Nat Rev Drug Discov 7, 591-607.

969 Xong, H.V., Vanhamme, L., Chamekh, M., Chimfwembe, C.E., Van Den Abbeele, J., Pays, 970 A., Van Meirvenne, N., Hamers, R., De Baetselier, P., Pays, E., 1998. A VSG expression 971 site-associated gene confers resistance to human serum in Trypanosoma rhodesiense. Cell $97295,839-846$.

973 
WT L. major
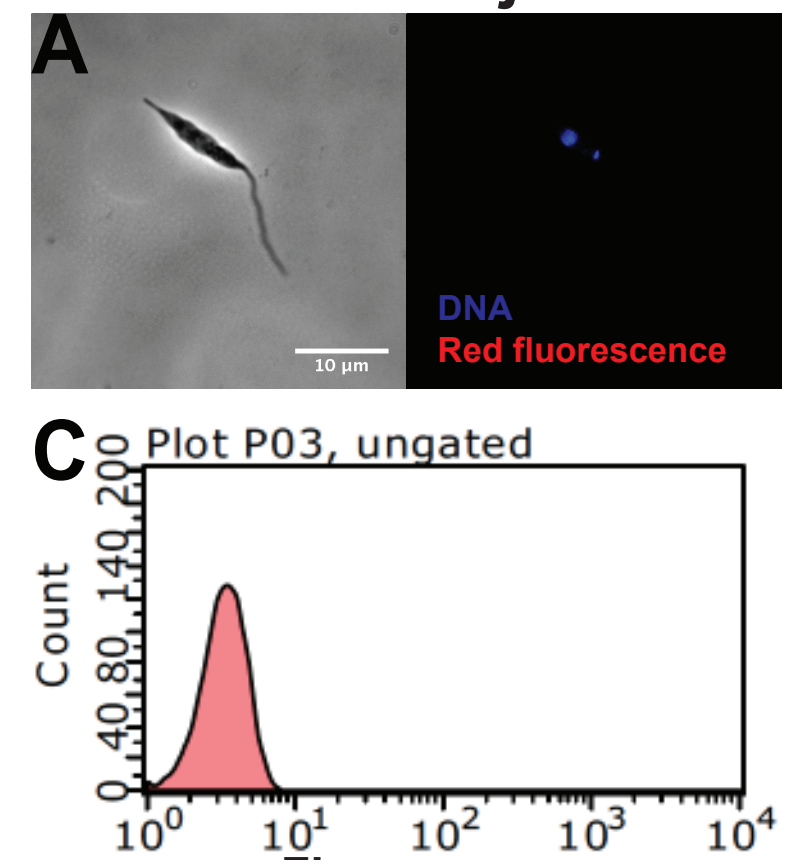
Fluorescence
RED L. major
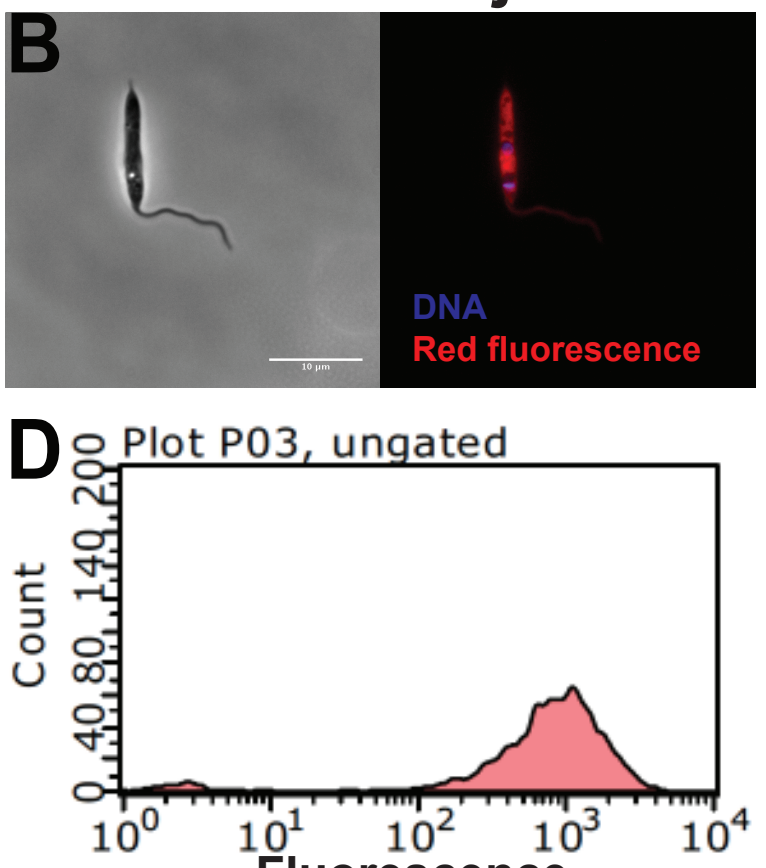

Fluorescence

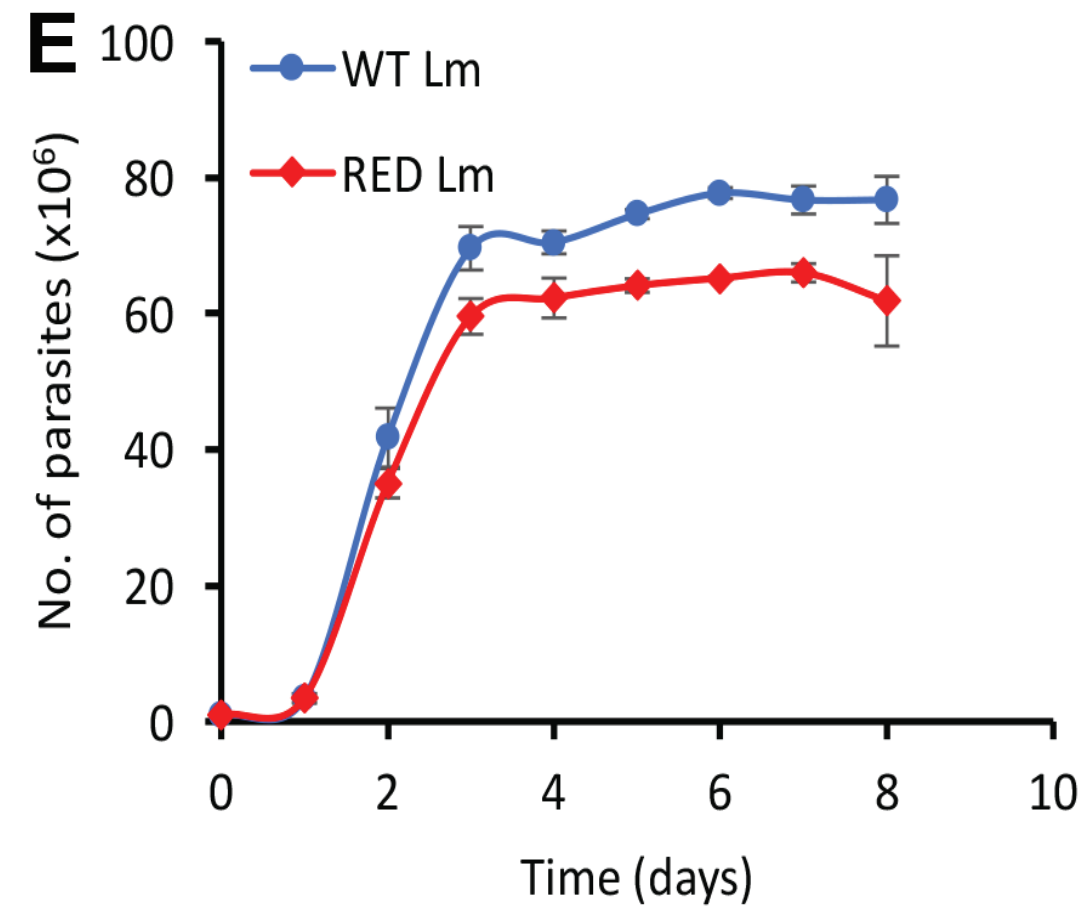


Bioluminescence

(Radiance in $\mathrm{p} / \mathrm{s} / \mathrm{cm}^{2} / \mathrm{sr}$ )
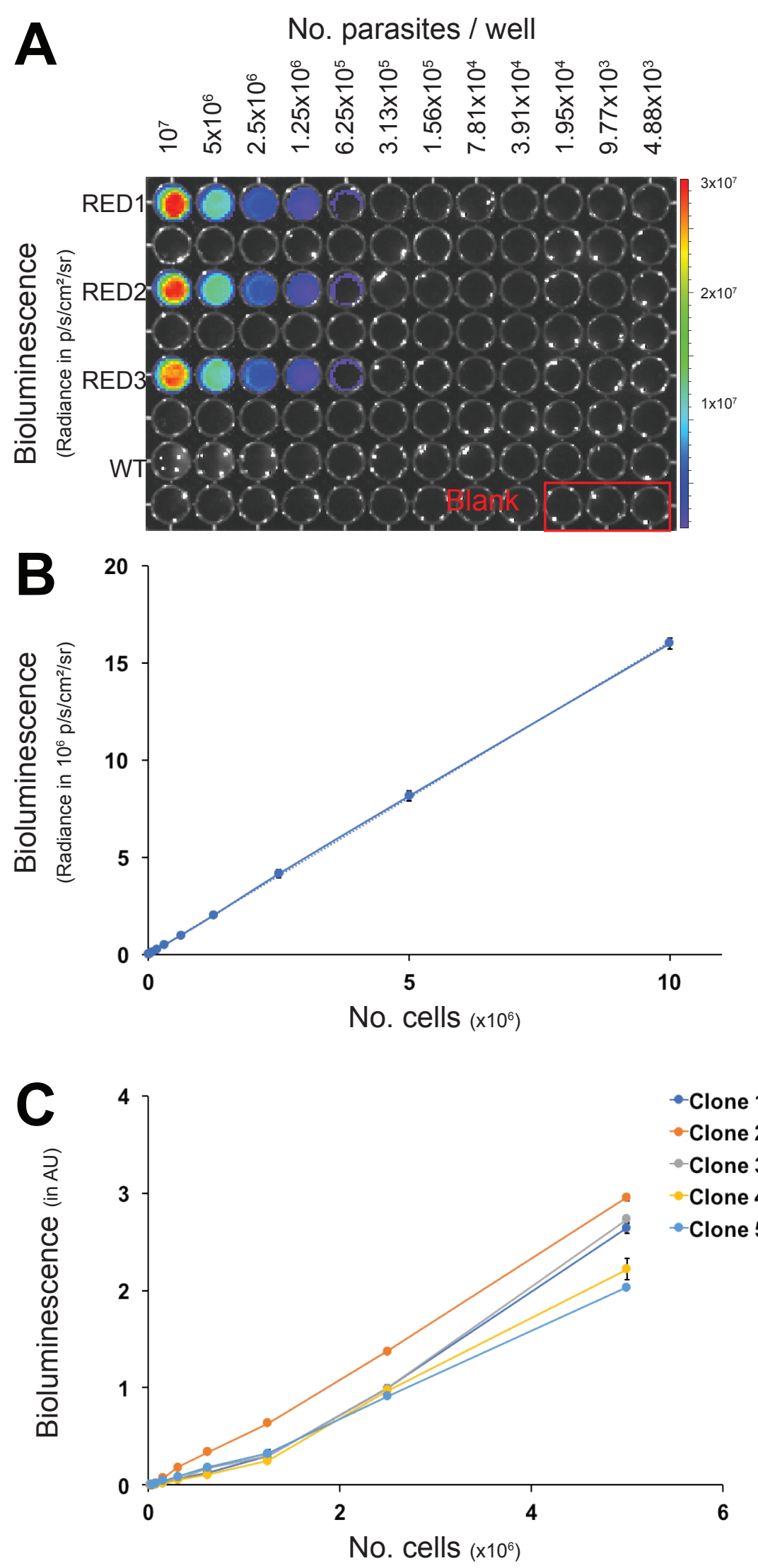

(Radiance in $\mathrm{p} / \mathrm{s} / \mathrm{cm}^{2} / \mathrm{sr}$ )
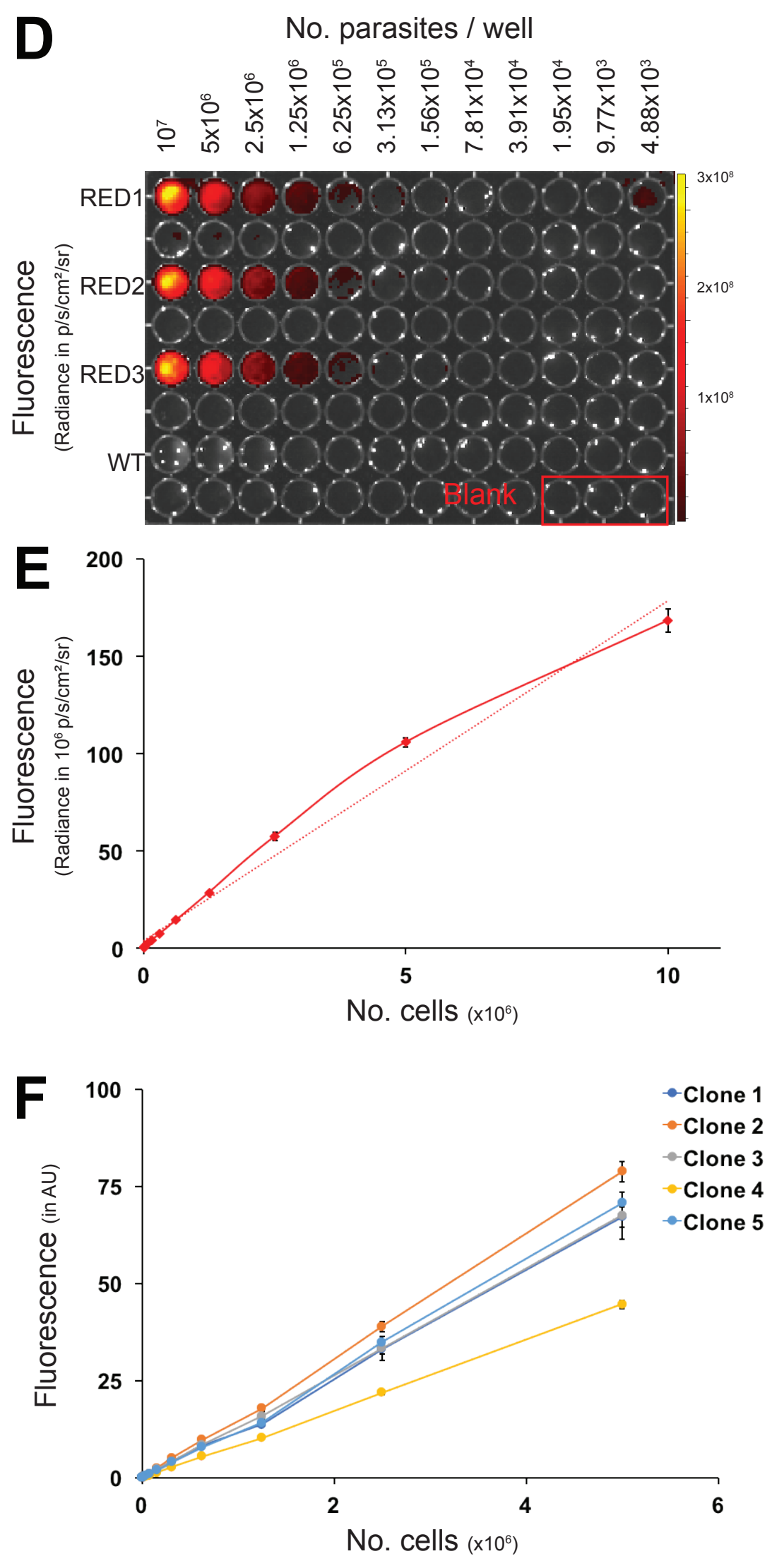


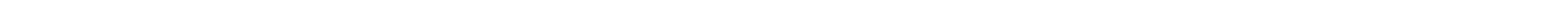


WT T. b. brucei
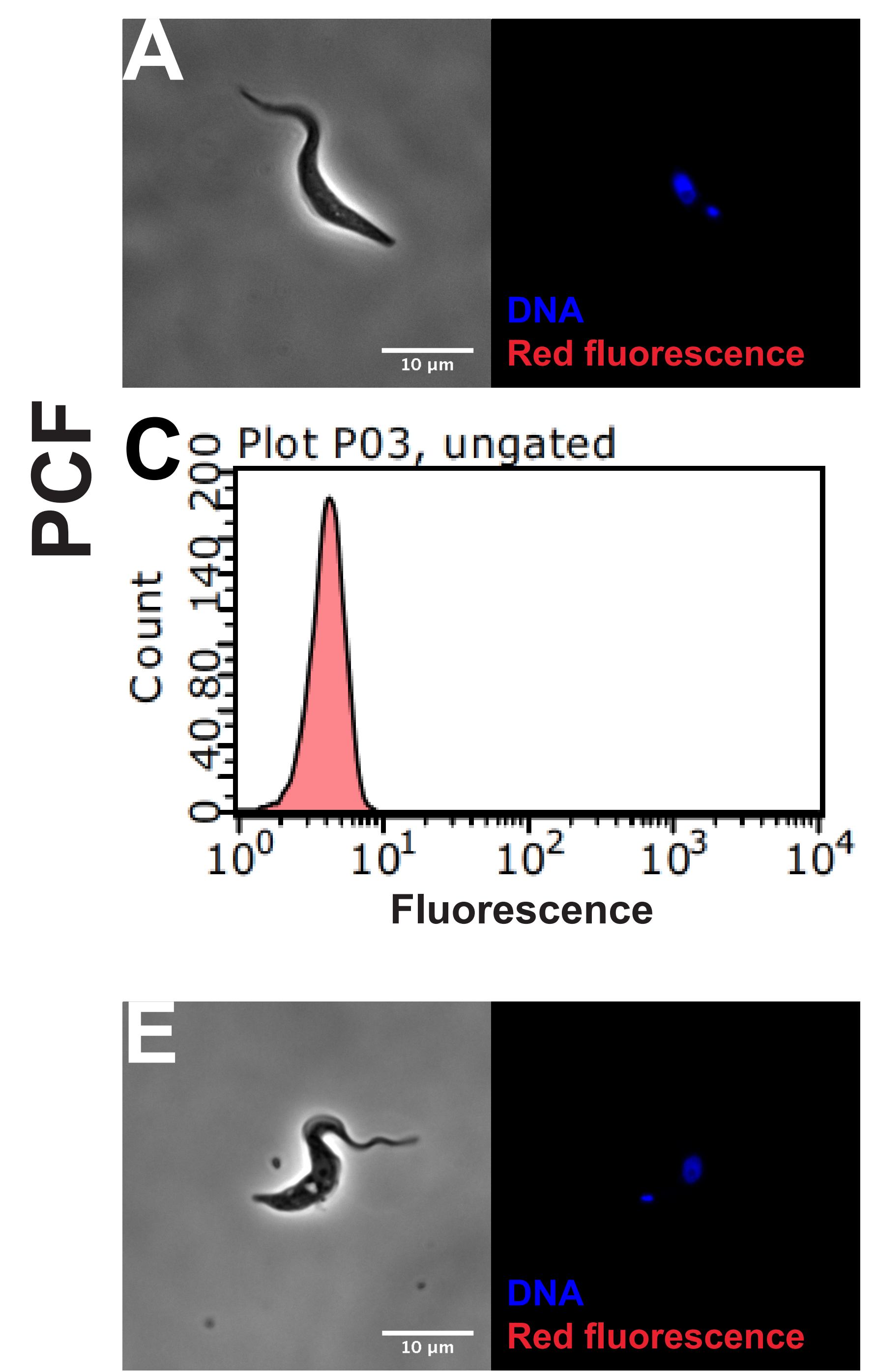

Go Plot P03, ungated

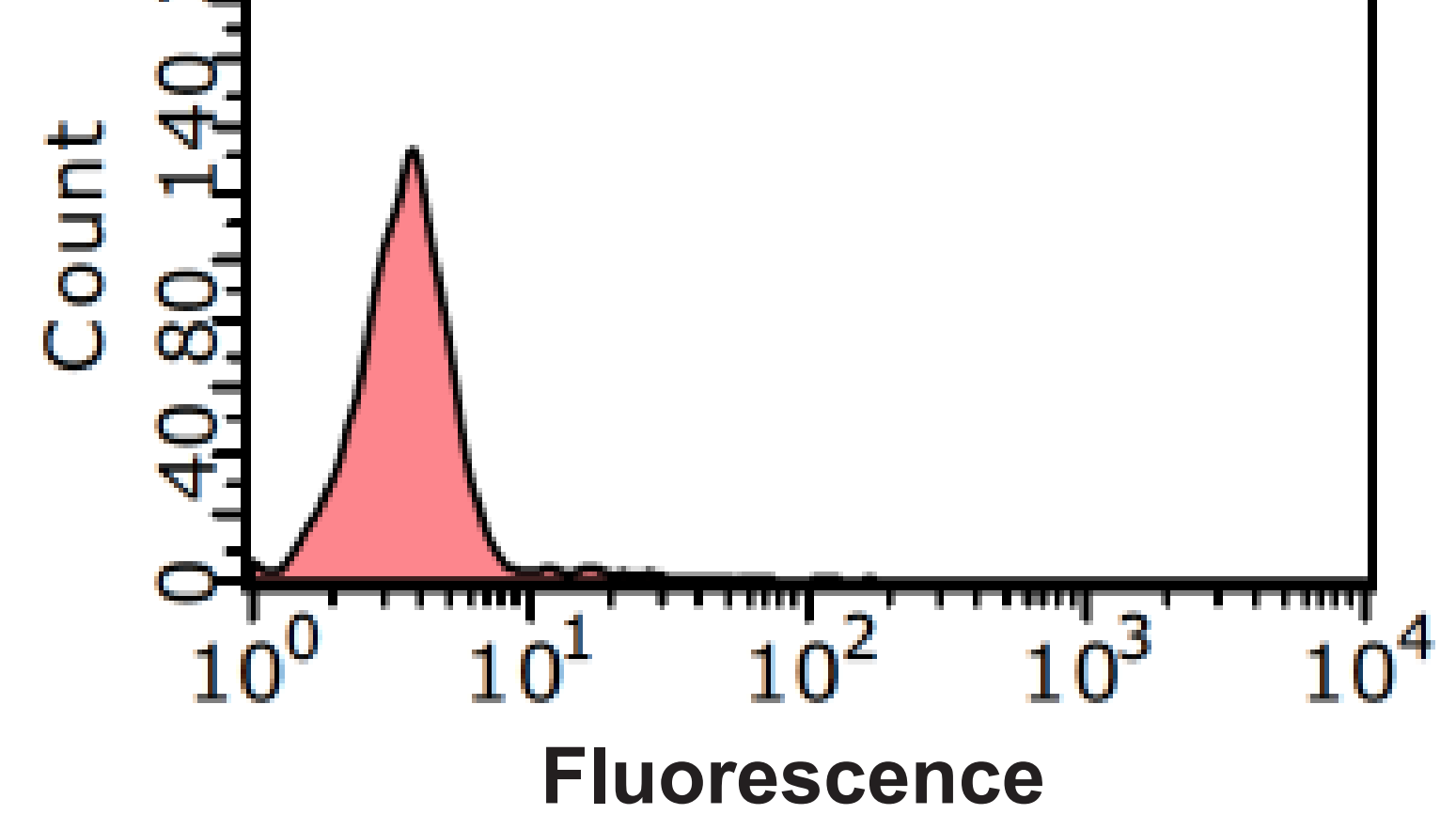

RED T. b. brucei
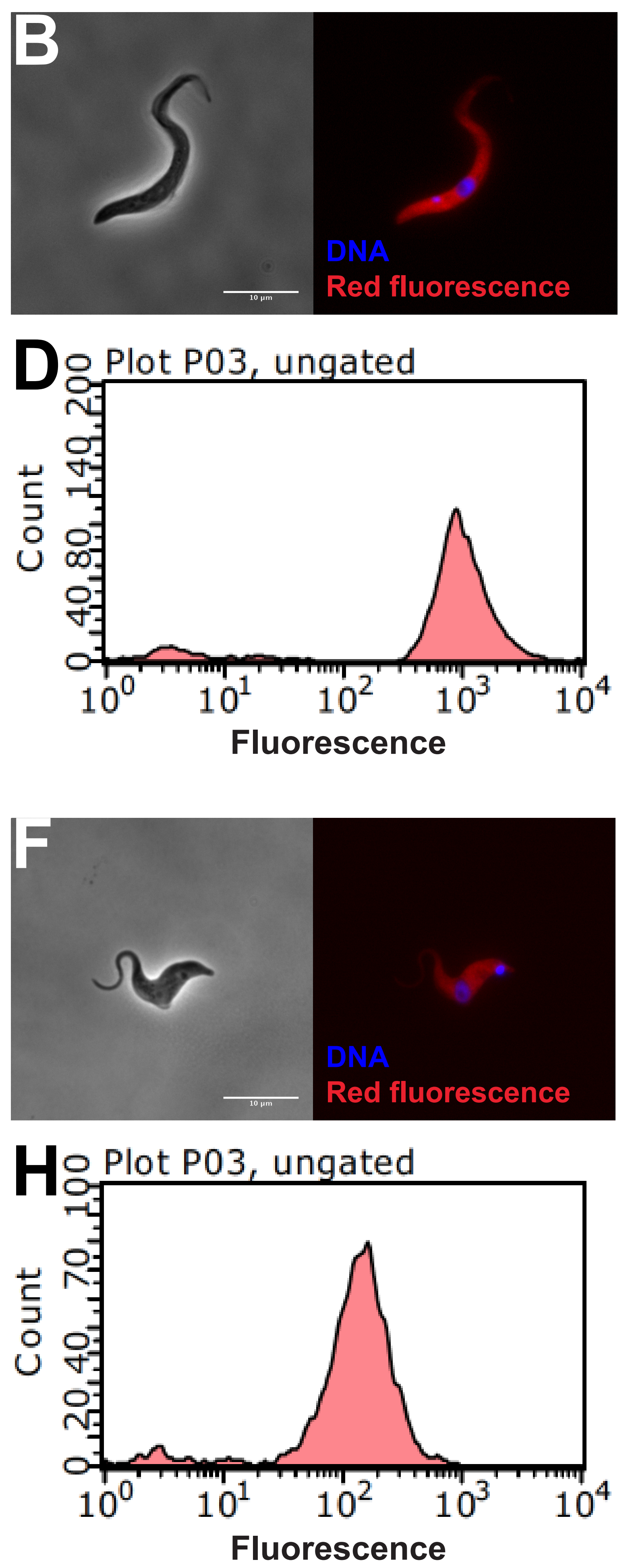
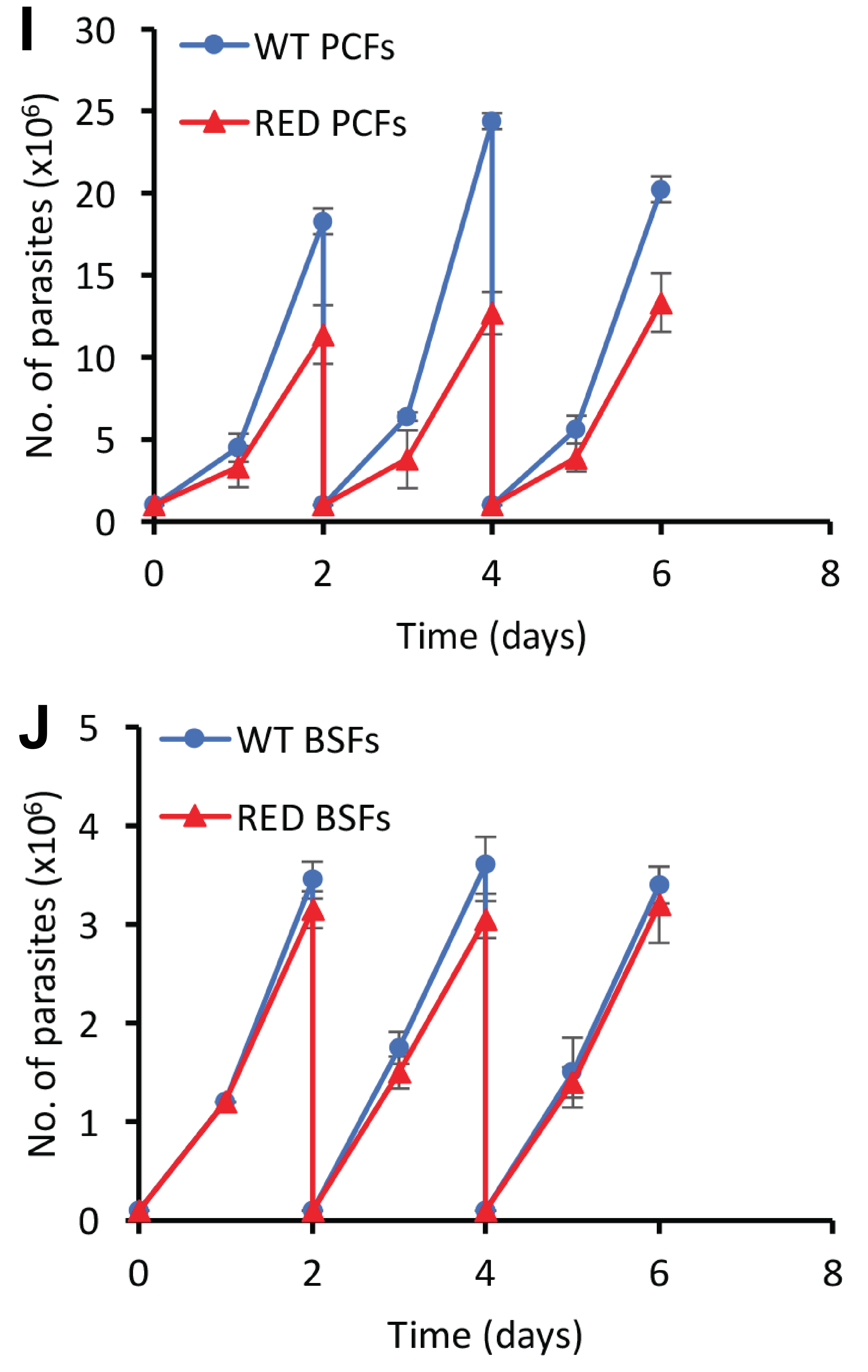
Bioluminescence

(Radiance in $\mathrm{p} / \mathrm{s} / \mathrm{cm}^{2} / \mathrm{sr}$ )

No. parasites / well

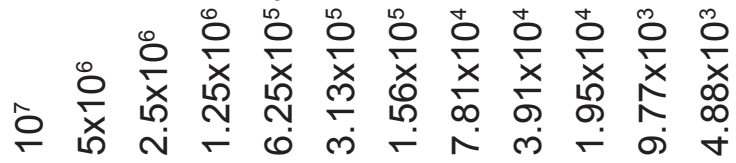

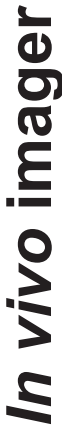

บ
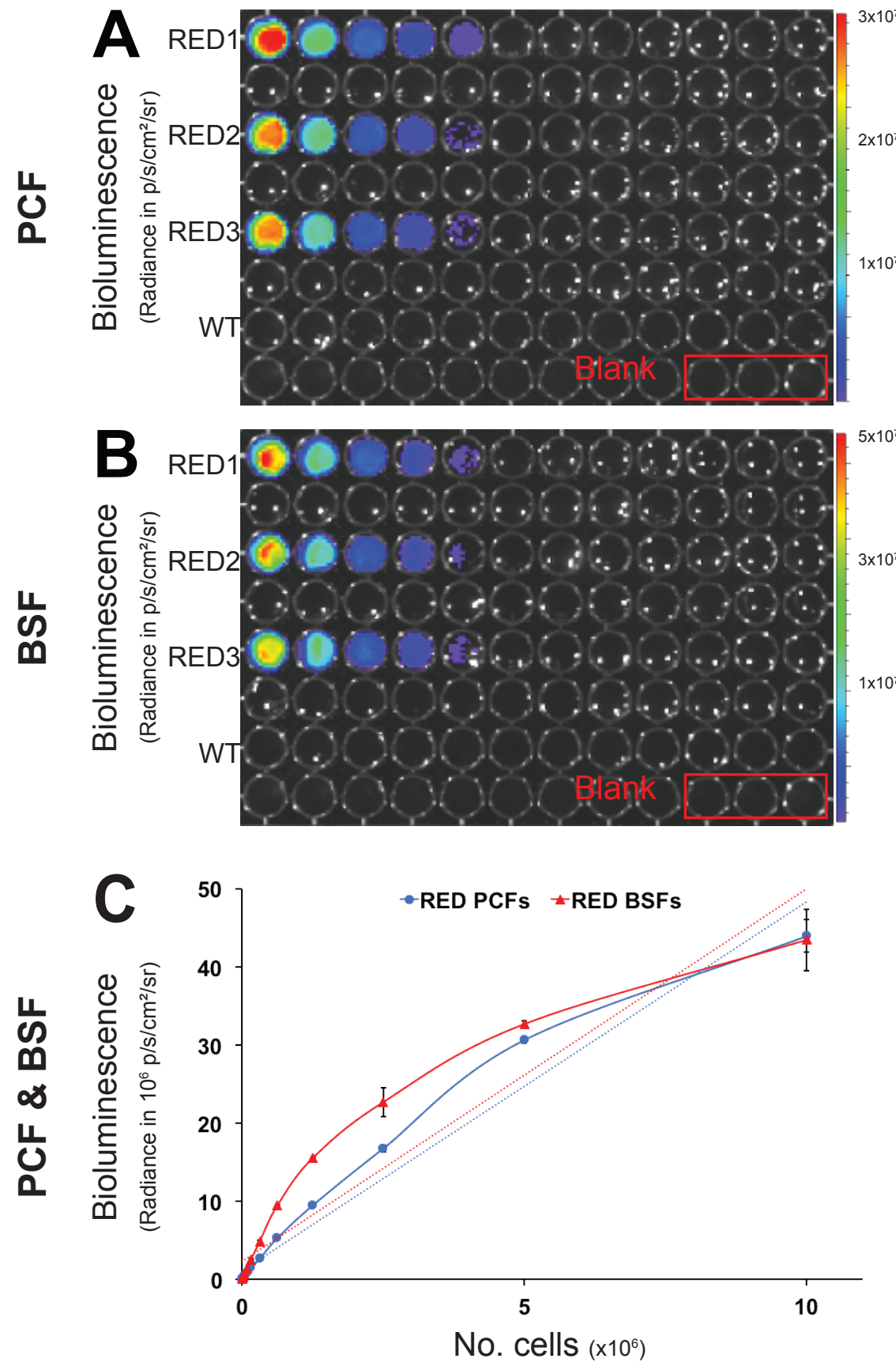

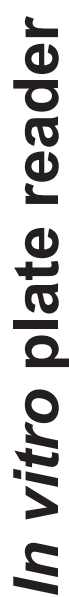
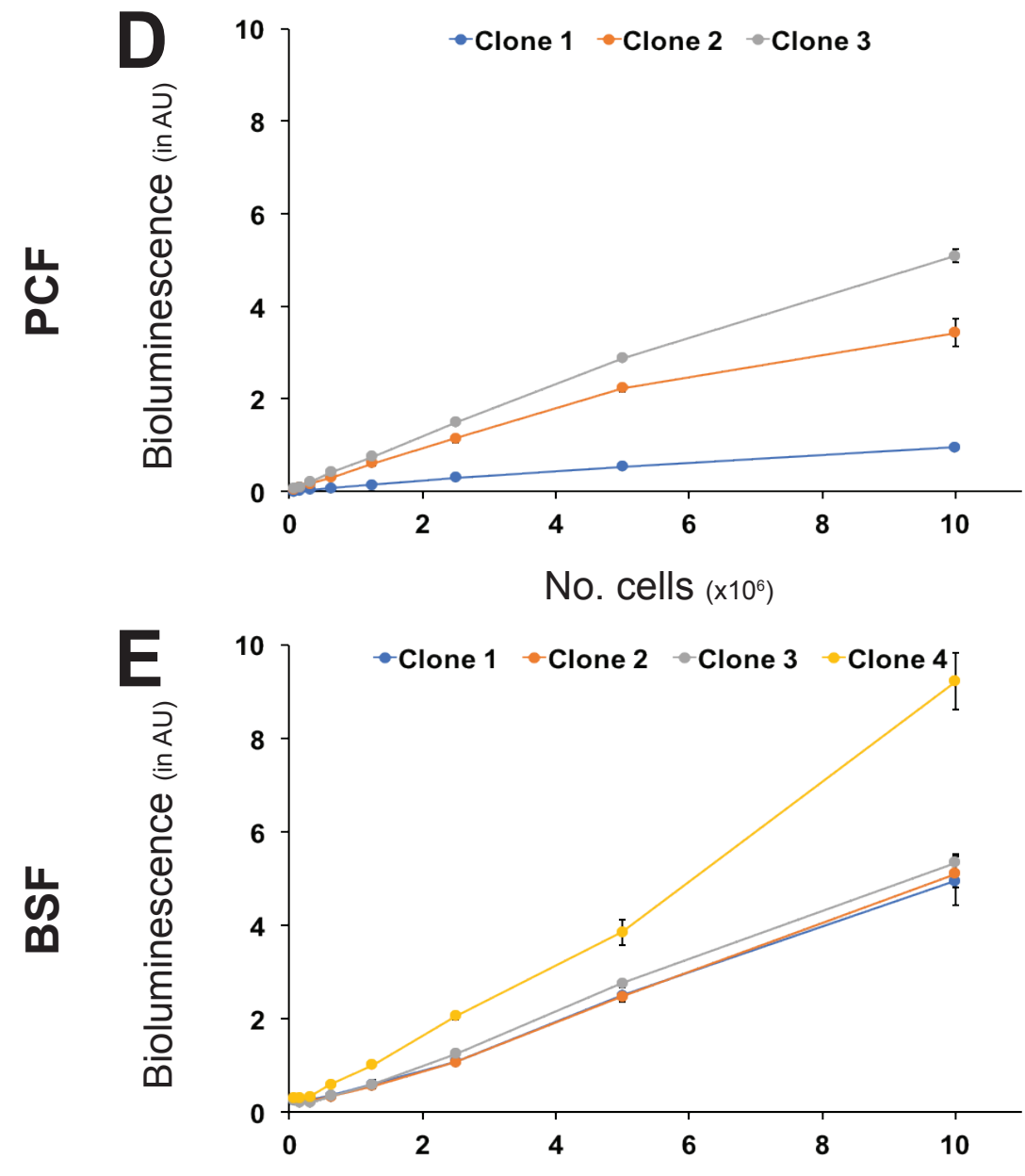

No. cells $\left(x 10^{6}\right)$
(Radiance in $\mathrm{p} / \mathrm{s} / \mathrm{cm}^{2} / \mathrm{sr}$ )

No. parasites / well
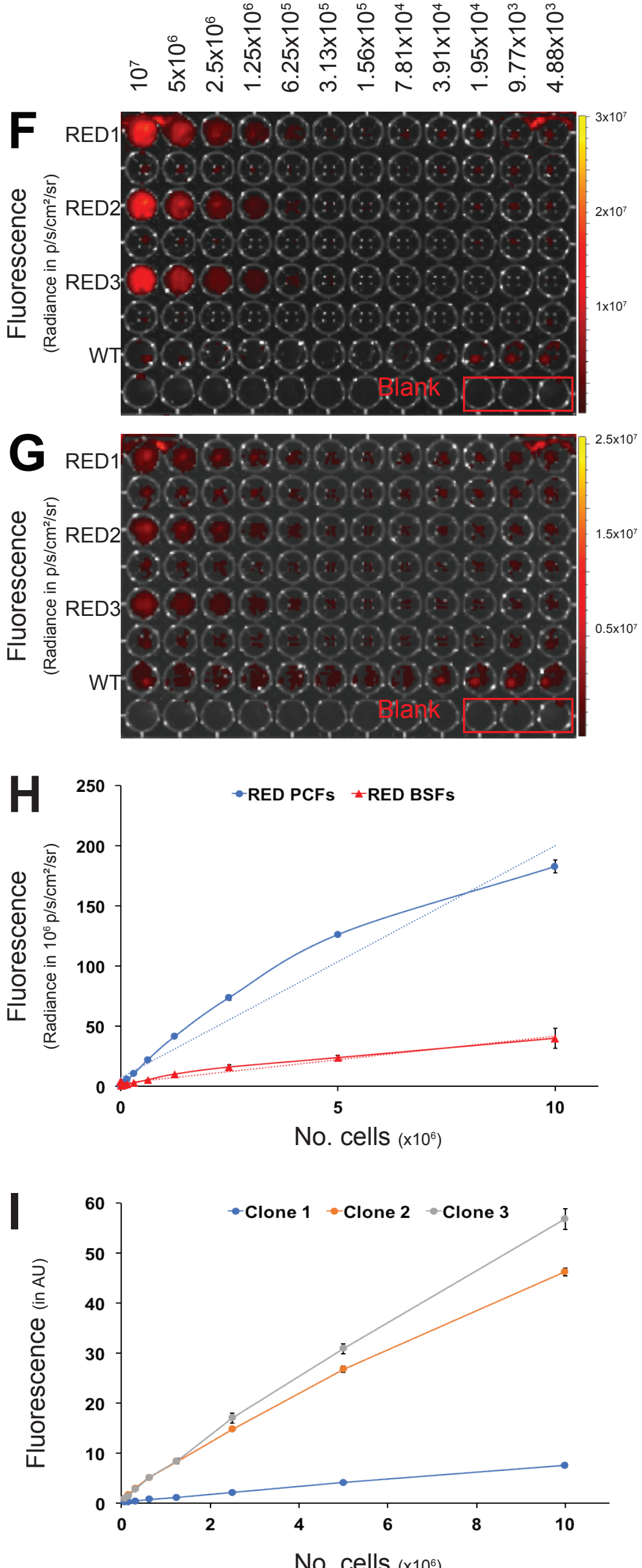

No. cells $\left(x 10^{6}\right)$

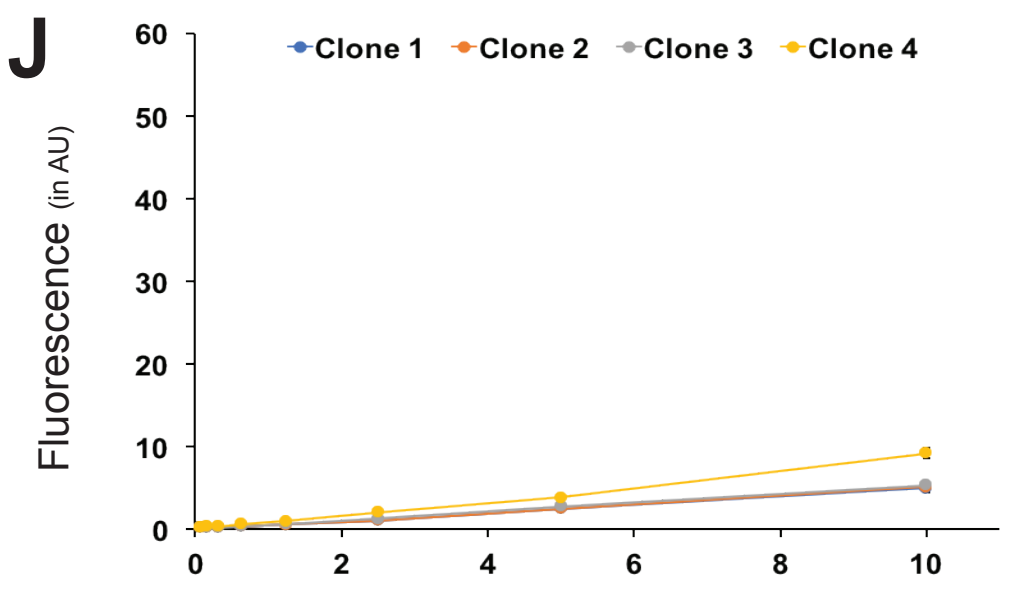

No. cells $\left(x 10^{6}\right)$ 


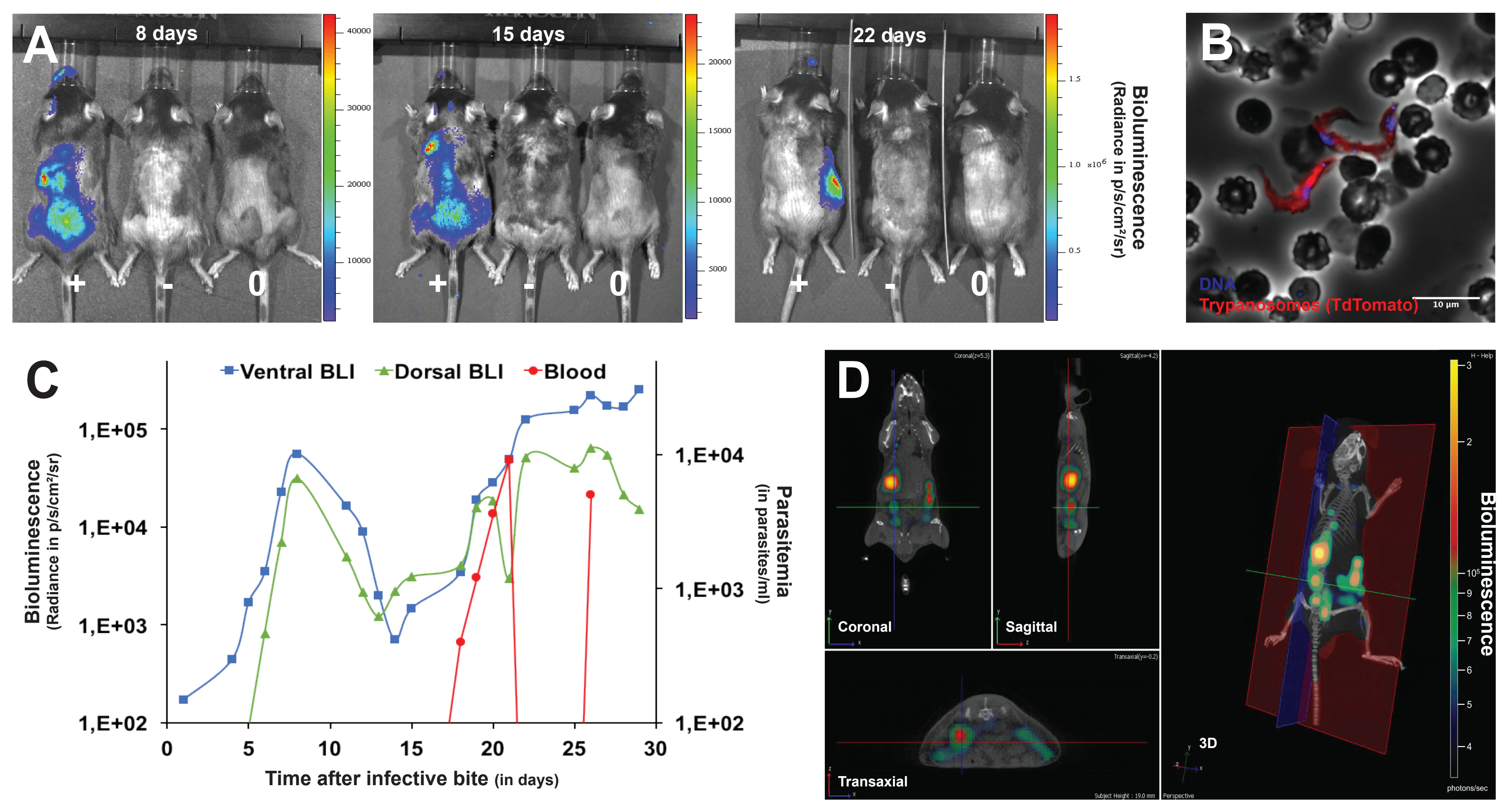




\section{A}

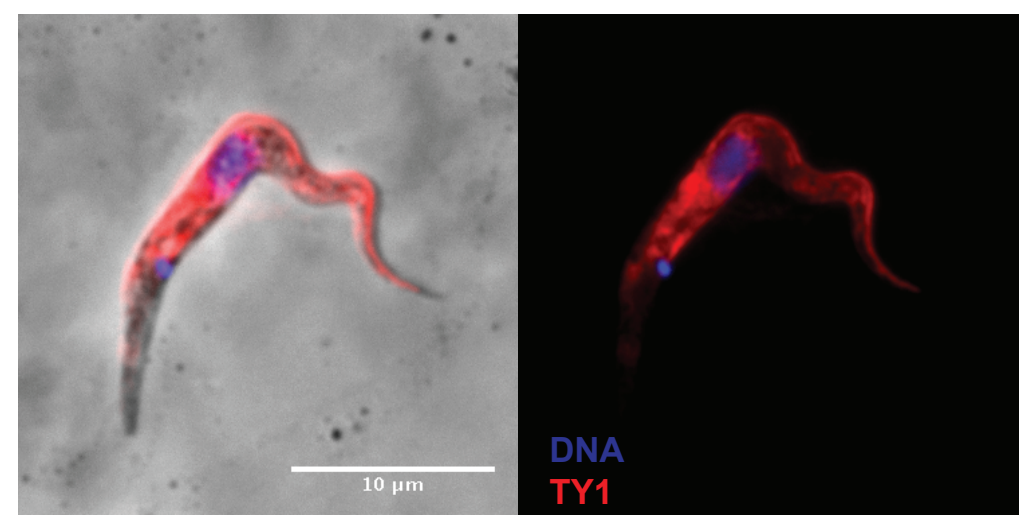

B

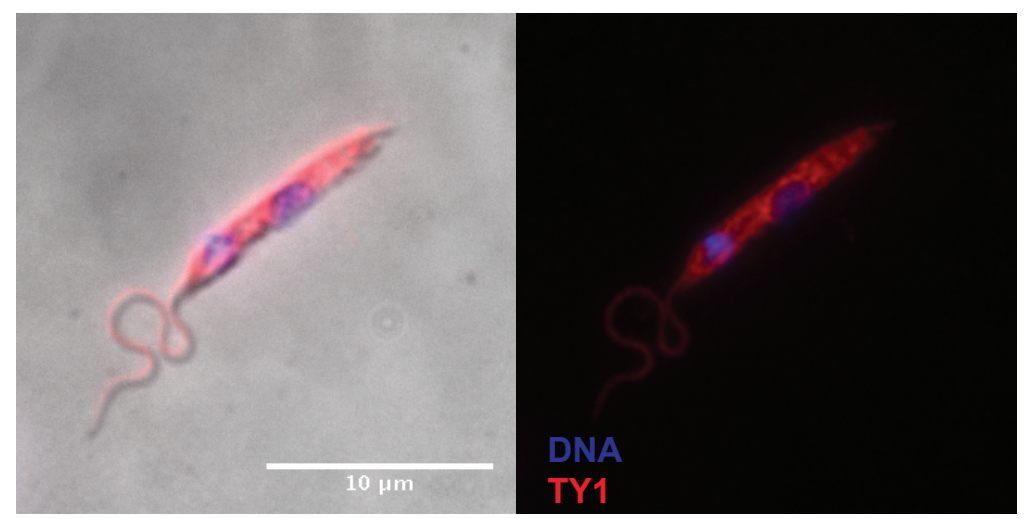

C

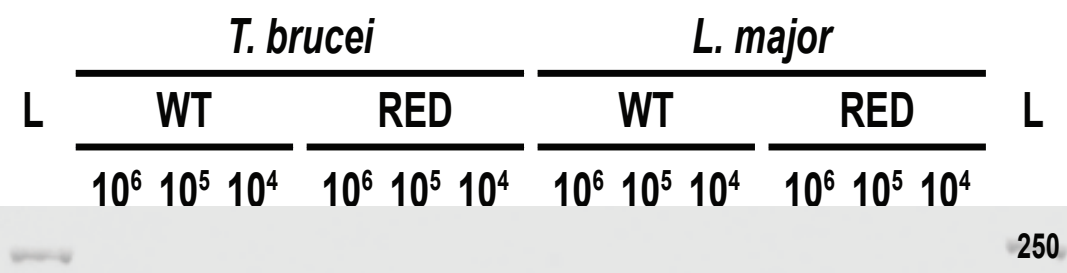

TY1

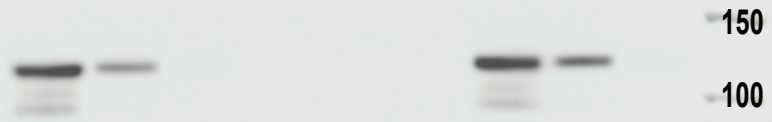

PFR

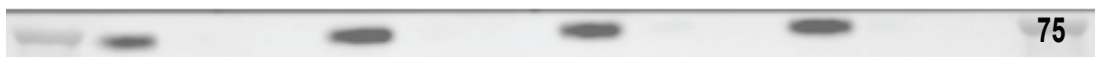

D

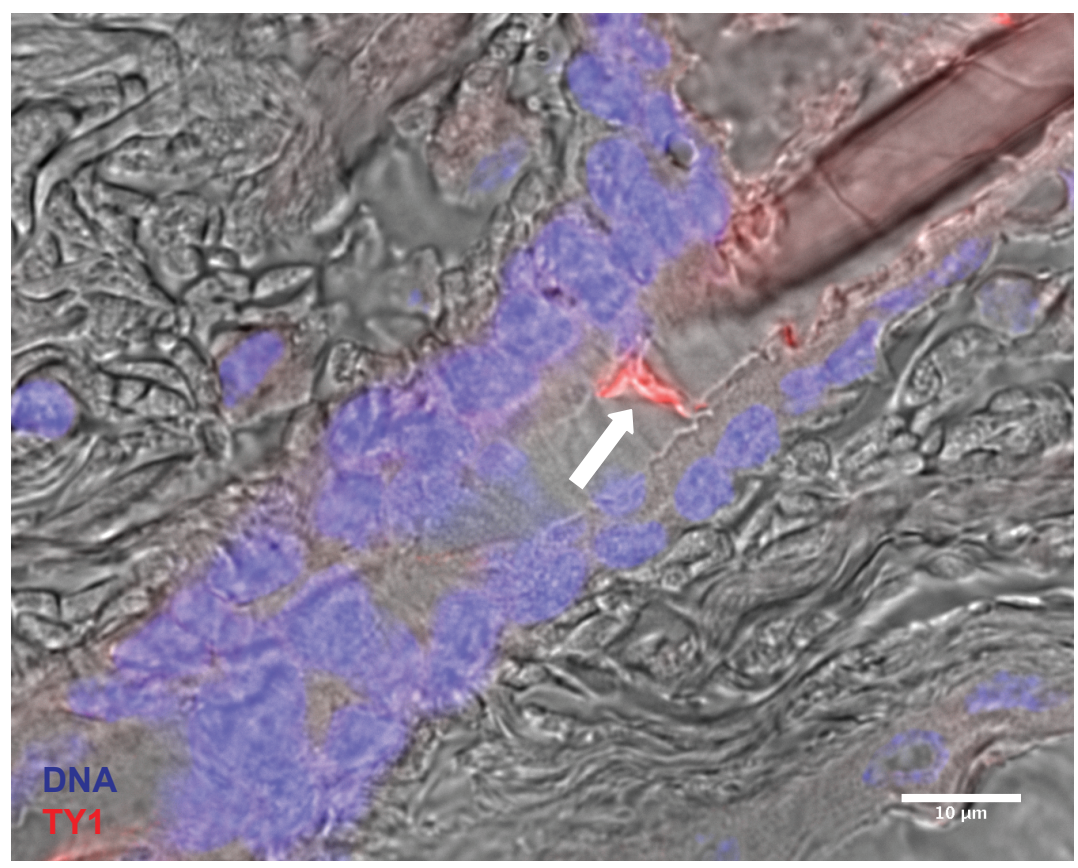


A

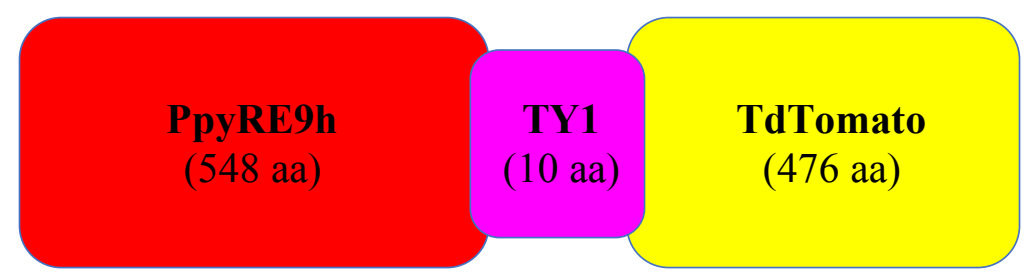

B

ATGGAGGACGCCAAGAACATCAAGAAGGGACCAGCCCCCTTCTACCCCCTGGAGGACGGCACAGCCGGCGAGCAGCTGCA CAAGGCCATGAAGCGGTACGCCCTGGTGCCAGGCACCATCGCCTTCACCGACGCCCACATCGAGGTGAACATCACCTACG CCGAGTACTTCGAGATGAGCGTGCGGCTGGCCGAGGCCATGAAGCGGTACGGCCTGAACACCAACCACCGGATCGTGGTG TGCAGCGAGAACAGCCTGCAGTTCTTCATGCCCGTGCTGGGAGCCCTGTTCATCGGCGTGGCCGTGGCCCCAGCCAACGA CATCTACAACGAGCGGGAGCTGCTGAACAGCATGAACATCAGCCAGCCCACCGTGGTGTTCGTGAGCAAGAAGGGCCTGC AGAAGATCCTGAATGTGCAGAAGAAGCTGCCCATCATCCAGAAGATCATCATCATGGACAGCAAGACCGATTACCAGGGC TTCCAGAGCATGTACACCTTCGTGACCAGCCACCTGCCCCCAGGCTTCAACGAGTACGACTTCGTGCCCGAGAGCTTCGA CCGGGACAAGACCATCGCCCTGATCATGAACAGCAGCGGCAGCACCGGCCTGCCCAAGGGCGTGGCCCTGCCCCACCGGG CCCTGTGCGTGCGGTTCAGCCACGCCAGAGACCCCATCTTCGGCAACCAGATCGCCCCCGACACCGCCATCCTGAGCGTG GTGCCCTTCCACCACGGCTTCGGCATGTTCACCACCCTGGGCTACCTGATCTGCGGCTTCCGGGTGGTGCTGATGTACAG GTTCGAGGAGGAGCTGTTCCTGCGGAGCCTGCAGGACTACAAGATCCAGACCGCCCTGCTGGTGCCCACCCTGTTCAGCT TCCTGGCCAAGAGCACCCTGATCGACAAGTACGACCTGAGCAACCTGCACGAGATCGCCTCTGGCGGAGCCCCACTGAGC AAGGAGGTGGGCGAGGCCGTGGCCAAGGGCTTCCACCTGCCAGGCATCCGGCAGGGCTACGGCCTGACCGAGACCACCAG CGCCATCCTGGTGACCCCCATCGGCGACGACAAGCCCGGAGCCGTGGGCAAGGTGGTGCCCTTCTTCGAGGCCAAGGTGG TGGACCTGGACACCGGCAAGACCCTGGGCGTGAACCAGAGAGGCGAGCTGTGCGTGAGAGGCCCCATGATCATGAGCGGC TACGTGAACAACCCCGAGGCCACCAACGCCCTGATCGACAAGGACGGCTGGCTGCACAGCGGCGACATCGCCTACTGGGA CGAGGACGAGCACTTCTTCATCGTGGACCGGCTGAAGAGCCTGATCAAGTACAAGGGCTACCAGGTGGCCCCAGCCGAGC TGGAGAGCATCCTGCTGCAGCACCCCAACATCCGGGACGCCGGAGTGGCCGGACTGCCCGACGACGACGCCGGAGAGCTG CCAGCCGCCGTGGTGGTGCTGGAGCACGGCAAGACCATGACCGAGAAGGAGATCGTGGACTACGTGGCCAGCCAGGTGAC CACCGCCAAGAAGCTGAGAGGCGGCGTGGTGTTCGTGGACGAGGTGCCCAAGGGCCTGACCGGCAAGCTGGACGCCAGAA AGATCCGGGAGATCCTGATCAAGGCCAAGAAGGGCGGCAAGATCGAGGTCCATACTAACCAGGACCCACTTGACaagctt ATGGTGAGCAAGGGCGAGGAGGTCATCAAAGAGTTCATGCGCTTCAAGGTGCGCATGGAGGGCTCCATGAACGGCCACGA GTTCGAGATCGAGGGCGAGGGCGAGGGCCGCCCCTACGAGGGCACCCAGACCGCCAAGCTGAAGGTGACCAAGGGCGGCC CCCTGCCCTTCGCCTGGGACATCCTGTCCCCCCAGTTCATGTACGGCTCCAAGGCGTACGTGAAGCACCCCGCCGACATC CCCGATTACAAGAAGCTGTCCTTCCCCGAGGGCTTCAAGTGGGAGCGCGTGATGAACTTCGAGGACGGCGGTCTGGTGAC CGTGACCCAGGACTCCTCCCTGCAGGACGGCACGCTGATCTACAAGGTGAAGATGCGCGGCACCAACTTCCCCCCCGACG GCCCCGTAATGCAGAAGAAGACCATGGGCTGGGAGGCCTCCACCGAGCGCCTGTACCCCCGCGACGGCGTGCTGAAGGGC GAGATCCACCAGGCCCTGAAGCTGAAGGACGGCGGCCACTACCTGGTGGAGTTCAAGACCATCTACATGGCCAAGAAGCC CGTGCAACTGCCCGGCTACTACTACGTGGACACCAAGCTGGACATCACCTCCCACAACGAGGACTACACCATCGTGGAAC AGTACGAGCGCTCCGAGGGCCGCCACCACCTGTTCCTGGGGCATGGCACCGGCAGCACCGGCAGCGGCAGCTCCGGCACC GCCTCCTCCGAGGACAACAACATGGCCGTCATCAAAGAGTTCATGCGCTTCAAGGTGCGCATGGAGGGCTCCATGAACGG CCACGAGTTCGAGATCGAGGGCGAGGGCGAGGGCCGCCCCTACGAGGGCACCCAGACCGCCAAGCTGAAGGTGACCAAGG GCGGCCCCCTGCCCTTCGCCTGGGACATCCTGTCCCCCCAGTTCATGTACGGCTCCAAGGCGTACGTGAAGCACCCCGCC GACATCCCCGATTACAAGAAGCTGTCCTTCCCCGAGGGCTTCAAGTGGGAGCGCGTGATGAACTTCGAGGACGGCGGTCT GGTGACCGTGACCCAGGACTCCTCCCTGCAGGACGGCACGCTGATCTACAAGGTGAAGATGCGCGGCACCAACTTCCCCC CCGACGGCCCCGTAATGCAGAAGAAGACCATGGGCTGGGAGGCCTCCACCGAGCGCCTGTACCCCCGCGACGGCGTGCTG AAGGGCGAGATCCACCAGGCCCTGAAGCTGAAGGACGGCGGCCACTACCTGGTGGAGTTCAAGACCATCTACATGGCCAA GAAGCCCGTGCAACTGCCCGGCTACTACTACGTGGACACCAAGCTGGACATCACCTCCCACAACGAGGACTACACCATCG TGGAACAGTACGAGCGCTCCGAGGGCCGCCACCACCTGTTCCTGTACGGCATGGACGAGCTGTACAAGTAA 
$5.0 \mathrm{~Kb}$

$4.0 \mathrm{~Kb}$

$3.0 \mathrm{~Kb}-$

$2.0 \mathrm{~Kb}-$

$1.0 \mathrm{~Kb}-$

$0.4 \mathrm{~Kb}-$

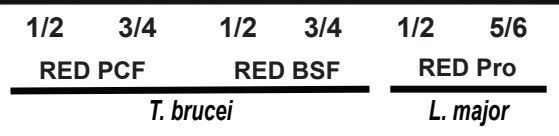

RED T. brucei

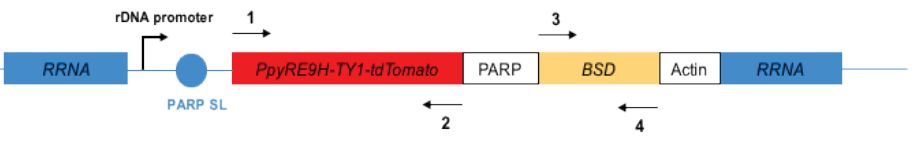

RED L. major

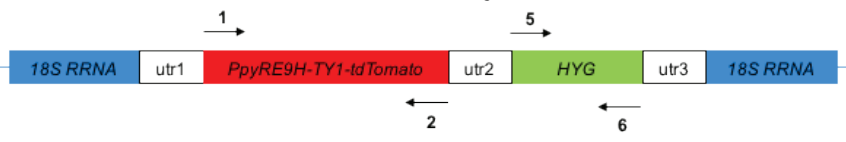




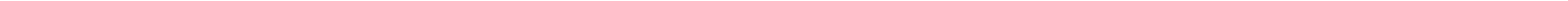

